\title{
Onset and stepwise extensions of recombination suppression are common in mating- type chromosomes of Microbotryum anther-smut fungi
}

Running title: Recombination cessation in MAT chromosomes

Marine Duhamel $^{1,2}$, Fantin Carpentier ${ }^{1}$, Dominik Begerow ${ }^{2}$, Michael E. Hood ${ }^{\# 3}$, Ricardo C.

5 Rodríguez de la Vega ${ }^{\# 1^{*}}$, Tatiana Giraud ${ }^{\# 1^{*}}$

\# These authors co-supervised the study; * Authors to whom correspondence should be addressed: Laboratoire Ecologie, Systématique et Evolution, Bâtiment 360, Université Paris-Saclay, 91400 Orsay, France. Phone: +33 1691556 69; fax: +33169154697

${ }^{1}$ Ecologie Systématique Evolution, Bâtiment 360, CNRS, AgroParisTech, Université Paris-Saclay, 91400

2

2 Evolution der Pflanzen und Pilze, Ruhr-Universität Bochum, Universitätsstraße 150, 44780, Bochum, Germany.

3 Department of Biology, Amherst College, 01002-5000 Amherst, Massachusetts, United State of America.

Key words: mating-type chromosomes, recombination suppression, fungi, sex chromosomes, mating-type loci, basidiomycetes, evolutionary strata, convergence

\begin{abstract}
Sex chromosomes and mating-type chromosomes can display large genomic regions without recombination. Recombination suppression often extended stepwise with time away from the sex- or mating-type-determining genes, generating evolutionary strata of differentiation

20 between alternative sex or mating-type chromosomes. In anther-smut fungi of the Microbotryum genus, recombination suppression evolved repeatedly, linking the two matingtype loci and extended multiple times in regions distal to the mating-type genes. Here, we obtained high-quality genome assemblies of alternative mating types for four Microbotryum fungi. We found an additional event of independent chromosomal rearrangements bringing

25 the two mating-type loci on the same chromosome followed by recombination suppression linking them. We also found, in a new clade analysed here, that recombination suppression
\end{abstract}


between the two mating-type loci occurred in several steps, with first an ancestral recombination suppression between one of the mating-type locus and its centromere; later, completion of recombination suppression up to the second mating-type locus occurred

30 independently in three species. The estimated dates of recombination suppression between the mating-type loci ranged from 0.15 to 3.58 million years ago. In total, this makes at least nine independent events of linkage between the mating-type loci across the Microbotryum genus. Several mating-type locus linkage events occurred through the same types of chromosomal rearrangements, where similar chromosome fissions at centromeres represent

35 convergence in the genomic changes leading to the phenotypic convergence. These findings further highlight Microbotryum fungi as excellent models to study the evolution of recombination suppression.

\section{Introduction}

Sex chromosomes in plants and animals can exhibit large genomic regions without

40 recombination (Bachtrog et al., 2014; Bergero and Charlesworth, 2009). Recombination suppression often extended away from sex-determining genes in a stepwise manner, generating discrete strata of genetic differentiation between alleles in the alternative sex chromosomes. Such evolutionary strata have long been thought to result from sexually antagonistic selection, i.e., the successive recruitment of genes with alleles beneficial only in one sex but harmful in the other, where selection favors complete linkage to the sexdetermining genes (Charlesworth, 2017). However, little evidence could be found in support of this hypothesis (Ironside, 2010) and recent studies have demonstrated the existence of recombination suppression across large regions and of multiple evolutionary strata on fungal mating-type chromosomes despite the lack of such antagonistic selection (Bazzicalupo et al.,

50 2019; Foulongne-Oriol et al., 2021; Hartmann et al., 2021; Menkis et al., 2008). Fungal mating-type chromosomes thus represent valuable emerging models for understanding how and why recombination suppression evolves (Hartmann et al., 2021).

Anther-smut fungi in the Microbotryum violaceum species complex (Basidiomycota) are particularly good models to address these questions. These plant-castrating fungi have an

55 obligate sexual cycle of meiosis and mating prior to infecting each new host, and they have megabase-long non-recombining regions on their mating-type chromosomes (Badouin et al., 2015; Branco et al., 2018, 2017; Carpentier et al., 2019; Hartmann et al., 2019; Hood et al., 
2004). This recombination suppression evolved independently at different times across the Microbotryum phylogeny, providing independent cases of evolution following recombination suppression with various ages (0.20-2.30 MY; Branco et al., 2018, 2017). Furthermore, in multiple Microbotryum species, recombination suppression has extended outward from the mating-type genes in several successive steps (Branco et al., 2018, 2017).

Most basidiomycete fungi carry two mating-type loci on different chromosomes and mating can only be successful between gametes harboring different alleles at both loci. The PR mating-type locus includes pheromone and pheromone receptor genes controlling gamete

65 fusion. The HD mating-type locus has two homeodomain genes controlling post-mating growth. In Microbotryum anther-smut fungi, the PR and HD loci were ancestrally located on different chromosomes (Branco et al., 2017). The different genes within each locus have been linked since the emergence of the basidiomycete clade (Coelho et al., 2017; Sun et al., 2019), and recombination suppression extended in the local region around each of the two mating-

70 type loci early in the diversification of Microbotryum fungi, generating independent evolutionary strata around the PR locus (called purple stratum) and HD locus (called blue stratum), and later another stratum (called orange) distal to the purple stratum (Branco et al., 2017). Subsequently, at least eight independent events of recombination suppression involving different chromosomal rearrangements linked the HD and PR loci (Branco et al.,

75 2018; Carpentier et al., 2021). The linkage of PR to HD mating-type determining genes is beneficial under the highly selfing system of Microbotryum fungi; only two mating types are then produced by a diploid individual, leading to a 1/2 compatibility among gametes under inter-tetrad selfing, i.e. among gametes produced by different meiosis events of a single diploid individual, and 2/3 compatibility under intra-tetrad selfing, i.e. among products of the very same meiosis. The linkage of the HD and PR mating-type loci thus doubles the odds of gamete compatibility under inter-tetrad selfing compared to a system with unlinked loci, in which a diploid individual produces four mating types, i.e. with 1/4 compatibility among gametes (Branco et al. 2017). In two Microbotryum fungi, the PR and HD loci became linked, not to each other, but to their respective centromeres; while this is not beneficial 85 under inter-tetrad selfing (1/4 compatibility), it yields the same 2/3 compatibility odds under intra-tetrad selfing as PR-HD linkage (Carpentier et al., 2019). In previous studies, we used the term "black strata” in referring all genomic regions linking the PR to HD mating-type loci to each other or to respective centromeres, although it should be reminded that they correspond to independent evolutionary events, trapping different sets of genes, with subsets 
90 overlapping among some of the black strata. HD-PR linkage was further followed, in several species, by stepwise extension of recombination suppression beyond the mating-type loci. The evolutionary and proximal causes for such extensions are still unknown (Hartmann et al., 2021); a recent theoretical model suggests that recombination suppression beyond matingtype loci may evolve due to the benefit of sheltering deleterious mutations (Jay et al., 2021).

95 The young evolutionary strata generated by these recombination suppression extensions in Microbotryum fungi were called by different colors in previous studies, e.g., white, light blue, pink, green and red (Branco et al., 2018).

The alternate alleles at genes in the non-recombining regions of mating-type chromosomes independently accumulate substitutions with time; their divergence is thus a proxy of time 100 since the cessation of recombination. For detecting distinct evolutionary strata, one typically uses non-synonymous substitutions $\left(\mathrm{d}_{\mathrm{s}}\right)$, i.e. not changing the amino-acid sequence, as they are considered neutral. In selfing species such as Microbotryum, being highly homozygous, the synonymous divergence between two alleles in recombining regions is null or very close to zero. The $d_{s}$ can therefore be used to delimit the non-recombining regions (NRRs) and the recombining pseudo-autosomal regions (PARs) of the mating-type chromosomes. Transspecific polymorphism is also used to study the age of recombination suppression linking genes to mating-type loci. Indeed, as soon as a gene is fully linked to a mating-type locus, its alternative alleles will remain associated to alternative mating types (called $a_{1}$ and $a_{2}$ in Microbotryum fungi), even across speciation events; in a genealogy, its alleles will therefore

110 be grouped according to mating type rather than according to species, and the nodes at which the alleles associated with the alternative mating types diverge indicate the time of recombination cessation (Branco et al., 2017, 2018; Hartmann et al., 2021).

In sex-determining homogametic/heterogametic systems, one chromosome still recombines, thus used as a proxy for the ancestral gene order (Lahn and Page, 1999). In contrast, because

115 mating types are determined at the haploid stage in fungi, both mating-type chromosomes are always heterozygous and both undergo frequent rearrangements in non-recombining regions, which renders the inference of historical steps of recombination suppression challenging. Previous studies have used as proxy of the ancestral state the chromosomal arrangement and gene order of an outgroup, $M$. intermedium, carrying its PR and HD loci on different 120 chromosomes and having its gene order highly syntenic with two other distantly related species, M. lagerheimii and M. saponariae, also carrying their PR and HD loci on different 
chromosomes (Branco et al., 2017; Carpentier et al. 2019). However, the genome of a single mating type of $M$. intermedium was available so far.

In this study, we therefore sequenced the genome of the lacking mating type of $M$. intermedium, as well as the haploid genomes of the two mating types of four additional

125 species displaying 2/3 compatibility between gametes within tetrads, suggesting either the linkage of the PR to the HD mating-type loci or the linkage of each mating-type locus to their respective centromeres (M.E. Hood, unpublished data). Among these four species, M. $v$. viscidula, M. v. gracilicaulis and M. v. parryi belong to clades with Microbotryum fungi carrying linked HD and PR mating-type loci, while $M$. v. lateriflora belongs to the clade with

130 the two species with HD and PR loci on distinct chromosomes, i.e., M. lagerheimii and M. saponariae. We analysed the organisation of the mating-type chromosomes in the new genomes sequenced in order to address the following questions: 1) Does $M . v$. lateriflora also carry its PR and HD mating-type loci on distinct chromosomes, with or without recombination suppression with the respective centromeres, or does it correspond to yet another independent event of HD-PR linkage? 2) Do the three other species with new genome sequenced display linked HD and PR loci, and do the phylogenetic placements and transspecific polymorphism indicate additional HD-PR linkage independent events from the previously documented ones? 3) In case of independent events of HD-PR linkage, did they occur through the same chromosomal rearrangements or yet new types of rearrangements? 4)

140 Are there footprints of evolutionary strata in the new genomes analysed, i.e., stepwise extension of recombination suppression?

\section{Material and methods}

\section{DNA extraction and sequencing}

DNA extraction and sequencing based on PacBio (Pacific Bioscience) long-read sequencing

145 was performed as described previously (Branco et al., 2018, 2017). Samples were collected before the publication of laws regarding the Nagoya protocol, if any, in the countries of collection. Microbotryum violaceum is a species complex, with most species being specialized on a single host species, especially when parasitizing Silene plant species (Hartmann et al., 2019; Le Gac et al., 2007a, 2007b). For the species of the complex that have 
commonly practiced for host races or formae speciales in phytopathology. For the present study, $a_{1}$ and $a_{2}$ haploid cells were isolated from a single tetrad from the following species: M. violaceum lateriflora parasitizing Moehringia lateriflora (strain 1509, Boothbay Harbor, Maine, USA, Coord.: 4351'34.8"N 69³7'45.6"W, collected in 2017), M. violaceum

155 gracilicaulis parasitizing Silene gracilicaulis (strain 1299, Wutoudi, Lijiang, China, Coord: $27^{\circ} 03^{\prime} 01.9^{\prime \prime} \mathrm{N} 100^{\circ} 11^{\prime} 36.5^{\prime \prime E}$, collected in 2014), M. violaceum parryi parasitizing S. parryi (strain 1510, Waterton lakes, Canada, Coord.: 49¹'48N 11350'24"W, collected in 2017), and M. violaceum viscidula parasitizing S. viscidula (strain 1506, Ninglang, Lijiang, China, Coord.: 27¹2'18.7" $100^{\circ} 47^{\prime} 35.7^{\prime \prime}$, collected in 2015). Cultivation of $\mathrm{a}_{1}$ and $\mathrm{a}_{2}$ haploid cells 160 was performed as in Le Gac al., 2007b. We sequenced the haploid genome of $M$. intermedium corresponding to the $\mathrm{a}_{2}$ mating type of the very same strain for which the haploid genome of the $\mathrm{a}_{1}$ mating type had already been sequenced (strain 1389-BM-12-12, collected on Salvia pratensis, Italy, Coord. GPS : 44²0'00.7"N 708'10.9"E; Branco et al. 2017).

\section{Genome assemblies and gene prediction}

Raw reads were processed using tools from the smrtanalysis suite 2.3.0 (https://github.com/PacificBiosciences/GenomicConsensus) as the previously published genomes (Badouin et al., 2015; Branco et al., 2018, 2017). We converted the bax.h5 files from the same sequencing run into one fastq file using pls2fasta. We generated the assembly 170 using canu v.1.8 (Koren et al., 2017) with the parameters "genomeSize=30m" and "-pacbioraw”. We used pbalign (version 0.3.0) with the blasr algorithm (Chaisson and Tesler, 2012) to realign the raw reads onto the assembly (indexed with samtools faidx; Li et al., 2009) and then used the output bam file into quiver (Chin et al., 2013) to polish the assembly basecalling. Default parameters were used when not detailed in the text. See Supplementary

175 Table S1 for assembly statistics.

As for the previously published Microbotryum high-quality genome assemblies (Badouin et al., 2015; Branco et al., 2018, 2017; Carpentier et al., 2019), the protein-coding gene models were predicted with EuGene 4.2a (Foissac et al., 2008), trained for Microbotryum. Similarities to the fungal subset of the uniprot database (Consortium TU, 2011) plus the $M$.

180 lychnidis-dioicae Lamole proteome (Badouin et al., 2015) were integrated into EuGene for the prediction of gene models.

\section{Transposable element detection and annotation}


De novo detection of transposable elements (TEs) was done using LTRharvest (Ellinghaus et al., 2008) from GenomeTools 1.5.10, performing long-terminal repeat (LTR) results from three other programs, RECON (Bao and Eddy, 2002), RepeatScout (Price et al., 2005) and Tandem Repeats Finder (Benson, 1999). The TE detection was enriched by BLASTn 2.6.0+ (Altschul et al., 1990) using the genomes as a database and the previously detected TE models as queries. To fulfill the repetitivity criterion, a TE sequence detected by

190 RepeatModeler or LTRHarvest and their BLAST hits were retained only if the query matched three or more sequences in the same species with an identity $\geq 0.8$, a sequence length $>$ 100bp and a coverage (defined as the query alignment length with removed gaps divided by the query length) $\geq 0.8$. When these criteria were met, the other query matches were retained for the following parameters: identity $\geq 0.8$, sequence length $>100 \mathrm{bp}$, e-value $\leq 5.3 \mathrm{e}-33$ and coverage $\geq 0.8$. TE annotation (Wicker et al., 2007) was performed using the fungal Repbase database 23.05 (Bao et al., 2015) based on sequence similarity. First, a BLASTn search and a BLASTx search were performed using the fungal Repbase DNA and protein sequence databases, respectively, and TE sequences as queries. For each of these similarity-based searches, the minimum e-value score was set at $1 e^{-10}$ and minimum identity at 0.8 . Then,

200 protein domain detection was performed using pfam_scan.pl (ftp://ftp.ebi.ac.uk/pub/databases/Pfam/Tools/) on TE sequences and compared to protein domain detection in the fungal Repbase database. Matches with e-values lower or equal to 1e-5 were kept. The annotation found by RepeatModeler was also considered. When no annotation was found, the TE sequence was annotated as "unclassified"; such annotations were discarded when overlapping with predicted genes. Annotation was performed using a python script. TE copies were removed from the predicted genes in further analyses.

\section{Comparative genomics}

Orthologous groups were obtained by Markov clustering (van Dongen, 2000) of high-scoring pairs parsed with orthAgogue (Ekseth et al., 2014) based on all-vs-all BLASTP 2.6.0

210 (Altschul et al., 1990) on protein sequences. The coding sequences (CDS) of 3,645 singlecopy genes present in all species analysed were aligned independently using MUSCLE (Edgar, 2004) as implemented in TranslatorX v1.1 (Abascal et al., 2010). We used IQ-TREE 2.0.4 (Minh et al., 2020) to build the maximum likelihood species tree with the TIM3+F model of substitution chosen according to the Akaike information criterion (AIC) by Model 215 Finder implemented in IQ-TREE (Kalyaanamoorthy et al., 2017). We used Rhodothorula 
babjavae as an outgroup. We assessed the robustness of the nodes with 1000 ultrafast bootstraps (Hoang et al., 2018; Minh et al., 2013) and SH-like approximate likelihood ratio tests (SH-aLRT) (Guindon et al., 2010) from the concatenated alignment. Pheromonereceptor (PR), pheromone and homeodomain (HD) genes were identified by BLASTN 2.6.0 220 (Altschul et al., 1990) similarity search using the pheromone receptor, pheromone and homeodomain gene sequences from M. lychnidis-dioicae (Devier et al., 2009; Petit et al., 2012). We identified the contigs belonging to the mating-type chromosomes for each species by i) identifying the contigs carrying $\mathrm{PR}$, pheromone and HD genes, ii) identifying the contigs carrying single-copy orthologous genes from the PR and HD chromosomes of $M$. intermedium, iii) identifying the contigs in the $\mathrm{a}_{1}$ (respectively $\mathrm{a}_{2}$ ) genome carrying singlecopy orthologous genes homologous to genes on the identified mating-type contigs of the $\mathrm{a}_{2}$ (respectively $\mathrm{a}_{1}$ ) genome, iv) plotting all these contigs using circos (http://circos.ca) comparing $a_{1}$ and $a_{2}$ genome to check which contigs really belong to the mating-type chromosomes (the contigs fully collinear between mating types and not assembled at an edge

230 of the mating-type chromosome were considered to belong to autosomal chromosomes, see Branco et al 2017). We oriented contigs in the direction involving the fewest inversions and keeping the PARs at the edges and non-inverted between mating-type chromosomes within a diploid genome. The contigs were considered joined when not broken at the same point in the alternative mating-type genomes within diploid individuals. Mating-type fission and fusion scenarios were inferred by linking orthologous genes on a circos plot between the matingtype contigs from one haploid genome with linked mating-type loci and the mating-type chromosomes of $M$. intermedium or $M$. lagerheimii, used as a proxy for the ancestral state as done previously (Branco et al., 2018, 2017).

Synonymous divergence $\left(d_{S}\right)$ was calculated from the alignment of $a_{1}$ and $a_{2}$ allele sequences 240 using MUSCLE (Edgar, 2004) implemented in TranslatorX v1.1 (Abascal et al., 2010). Synonymous divergence $\left(\mathrm{d}_{\mathrm{s}}\right)$ and its standard error were calculated using the yn00 v4.9f program of the PAML package (Yang, 2007) and plotted using the ggplot2 library of $\mathrm{R}$ (Wickham, 2009). Non-recombining regions were identified as non-collinear regions with non-null synonymous divergence using the $\mathrm{a}_{1}-\mathrm{vs}-\mathrm{a}_{2}$ circos and $\mathrm{d}_{\mathrm{s}}$ plots. Pseudo-autosomal 245 regions were identified as the remaining regions, being collinear and with null synonymous divergence, as expected in these highly selfing fungi. 
Centromeres (Supplementary Table S2) were identified by blasting (BLASTN 2.6.0 Altschul et al., 1990) the centromeric repeats previously described (Badouin et al., 2015). Centromeres were defined as stretches of centromeric repeats (Supplementary Table S3), two consecutive repeats being distant by maximum 50kb; only the largest block was kept when several stretches of centromeric repeats were found on the same contig. Only contigs larger than 20kb were considered. The delimitation of putative centromeres was recursively extended by $1 \mathrm{~kb}$ windows as long as gene density was lower than 0.25 in the focal window. Results were congruent with previously identified centromeres (Branco et al., 2018). Telomeres

255 (Supplementary Table S4) were delimited in the 100bp region at the end of contigs carrying at least five times the telomere-specific TTAGGG motif (CCCTAA on reverse complementary strand) as done previously (Badouin et al., 2015).

\section{Trans-specific polymorphism and datation}

We performed codon-based alignment with macse v2.0 (Ranwez et al., 2018) of one-to-one

260 orthologs of genes ancestrally located between the PR locus and its centromere (23 genes), between this centromere and the short arm telomere (11 genes), between the HD locus and its centromere (8 genes) or in the light blue $M$. caroliniana stratum (14 genes). Gene trees were obtained with IQ-TREE 2.0.4 (Minh et al., 2020) with automatic selection of the substitution model (Kalyaanamoorthy et al., 2017) and branch-support estimated with 1000 ultrafast

265 bootstraps (Hoang et al., 2018; Minh et al., 2013). Tree topology congruence was assessed with the approximate unbiased (AU) test (Shimodaira, 2002) with 10,000 RELL replicates (Kishino et al., 1990) implemented in IQ-TREE 2.0.4 (-zb 10000 and -au options). For the genes ancestrally located between the PR locus and its centromere, between the centromere of the ancestral PR chromosome and its short arm telomere and between the HD locus and its centromere we tested whether each gene alignment was significantly conflicting with each of the different topologies obtained among the 41 other gene trees, using the $-\mathrm{z}$ option and the substitution model fixed to the one found during the focal gene tree reconstruction. We also compared the topology of the species tree and the tree obtained by concatenating the conserved genes in the region proximal to the PR locus corresponding to light blue and red

275 strata. Trees with pAU values greater than 0.05 were considered non-discordant. The codonbased multiple alignments were concatenated separately for these regions, producing alignments of 19,944 (PR locus to centromere), 3,955 (centromere ancestral PR to short-arm telomere), 7,705 (HD locus to centromere) and 9,952 (proximal region of the PR locus on the long arm side, corresponding to the light blue and red strata) codons. Partitionated alignments 
280 (one partition per codon position) were imported to BEAUti v2.5.0 (Bouckaert et al., 2014) to produce BEAST2 xml input files with the following priors: unlinked gamma site model with 3 categories and HKY substitution model; strict clock and gamma clock rate with alpha 0.001 and beta 1,000; calibrated Yule tree model with gamma birth alpha 0.001 and beta 1,000; exponential gamma shape per partition; log-normal HKY’s kappa. A calibration point at each

285 of the two divergence nodes between M. lychnidis-dioicae and M. silenes-dioicae, one node separating the $\mathrm{a}_{1}$-associated alleles and the other node the $\mathrm{a}_{2}$-associated alleles, both set as normally distributed with mean 0.42 MY and sigma 0.04. BEAST2 v2.4.6 (Bouckaert et al., 2014); runs were performed for 20,000,000 iterations and written every 1,000 iterations. Date estimates were obtained with the 15,000 trees with best posterior probabilities.

\section{Results}

We obtained high-quality assemblies (Supplementary Table S1) for a genome of the previously unavailable haploid mating type $\left(\mathrm{a}_{2}\right)$ of $M$. intermedium, as well as the haploid genomes of both mating types of four additional species compared to earlier studies: $M$. violaceum lateriflora parasitizing Moehringia lateriflora, M. violaceum gracilicaulis parasitizing Silene gracilicaulis, $M$. violaceum parryi parasitizing $S$. parryi, and $M$. violaceum viscidula parasitizing $S$. viscidula. The species tree obtained based on 3,645 single-copy genes was robust and congruent with previous studies; the new species were distributed across the phylogeny (Figure 1).

\section{Ancestral state in Microbotryum intermedium}

300 The assembly of the $\mathrm{a}_{2} M$. intermedium genome recovered full $\mathrm{HD}$ and PR mating-type chromosomes, with telomeric repeats at the ends of the contigs and well-defined centromeres, thus confirming that the PR and HD loci are unlinked in this species. We found, as in previous studies on other Microbotryum species (Branco et al. 2017, 2018), that autosomes were completely collinear between the two haploid genomes of the sequenced $M$. intermedium diploid strain (Supplementary Figure S1A), and autosomal genes had zero $\mathrm{d}_{\mathrm{S}}$ levels between the allele copies (Supplementary Figure S2A); $d_{\mathrm{S}}$ levels and collinearity are therefore good indicators of the occurrence of recombination and these selfing species are highly homozygous. By comparing the alternative mating-type chromosomes of $M$. intermedium, we detected few rearrangements (Figure 2A) and the $d_{s}$ plots mostly displayed 
310 zero values, as observed in autosomes (Supplementary Figures S1A and S2A). These observations confirm that there is likely no recombination suppression along most of the $M$. intermedium mating-type chromosomes. High $\mathrm{d}_{\mathrm{s}}$ values and rearrangements were only observed in proximity to the PR locus, indicating that this region does not recombine, corresponding to the stratum called "purple" that was previously identified in the other

315 Microbotryum species (Figure 3A). This purple evolutionary stratum thus likely evolved before the divergence of $M$. intermedium from the other Microbotryum species (Figure 1).

We found a small inversion near the centromere in the PR mating-type chromosome that distinguished $M$. intermedium from another species also with PR and HD loci retained on separate mating-type chromosomes, M. lagerheimii (Figure 2B). Because the M. lagerheimii arrangement was found in all the Microbotryum species analysed except $M$. intermedium and M. scabiosae (Figure 2B and Supplementary Figure S3O), we inferred that M. intermedium harbored the ancestral gene order and that the inversion around the PR centromere occurred early in the Microbotryum clade, after the divergence of the rest of the Microbotryum clade from the $M$. scabiosae lineage (Figure 1). We therefore consider the $M$. lagerheimii gene order as ancestral for inferring chromosomal rearrangement scenarios in the lineages that have derived subsequent to this inversion near the PR chromosome centromere (Figure 1).

\section{Discovery of additional independent events of mating-type loci linkage}

The evolutionary history of mating-type chromosomes was inferred in four Microbotryum species studied for the first time here (Figure 1, M. v. gracilicaulis, M. v. viscidula, M. v.

330 lateriflora and M. v. parryi) by comparing their genomes to the M. lagerheimii genome. We obtained high-quality assemblies of two haploid genomes of opposite mating types per species, i.e. haploid genomes of alternative mating types $\left(\mathrm{a}_{1}\right.$ and $\left.\mathrm{a}_{2}\right)$ produced by meiosis of a single diploid individual. The mating-type chromosomes were assembled in few contigs, not interrupted at the same places in the two mating-type chromosomes in each species (Figure 2 and Supplementary Figure S4), which allowed complete joining of contigs. The PR and the HD loci were found on a single contig in at least one haploid genome of each species, indicating that these species all have their mating-type loci linked together on a single chromosome (Figures 2C and 2E; Supplementary Figures S4A and S4C). The pseudoautosomal regions were defined as fully collinear regions lacking synonymous divergence

340 between alleles (i.e. with the same patterns as in autosomes; Figure 2; Supplementary Figures 1 and 2). 
We reconstructed the evolutionary history of their mating-type chromosomes by comparing their genome structures to those of $M$. lagerheimii, taken as a proxy for their genomic ancestral state before chromosomal fusion events. The mating-type chromosome fissions

345 (always found at centromeres) were determined by assessing, in synteny plots (e.g. Figure 2 and Supplementary Figure S4), what arms of the ancestral mating type chromosomes became autosomes, i.e. were completely collinear between mating-types and assembled separately from the derived mating-type chromosomes in both haploid genomes. The orientation of ancestral mating-type chromosome fusion was assessed by determining on synteny plots (e.g.

350 Figure 2 and Supplementary Figure S4) what edge of ancestral mating-type chromosomes remained recombining, i.e. became PARs, the other edge of the ancestral chromosome or the centromere thus corresponding to the fusion point. For orienting the few contigs within the non-recombining regions that could not be assembled with the PAR contigs, we applied a majority rule minimizing inversion numbers with the alternative mating type; their orientations do not impact the scenario reconstruction.

In $M . v$. gracilicaulis parasitizing Silene gracilicaulis (Figure 2D) and $M . v$. viscidula parasitizing Silene viscidula (Supplementary Figure S3B), mating-type locus linkage was achieved through the fusion of the entire PR chromosome and the short arm of the HD chromosome. This represents the same chromosomal rearrangement as in M. lychnidisdioicae, M. silenes-dioicae, M. coronariae, M. violaceum s.s. and M. v. melanantha. The placement of these species as a clade in the phylogeny suggests that this fission/fusion event can represent an ancestral rearrangement to this clade (Figure 1). Under the alternative hypothesis, the same rearrangement would have occurred several times independently.

In order to test the independence of the mating-type locus linkage events in this clade, we analysed trans-specific polymorphism by reconstructing the evolutionary history of $\mathrm{a}_{1}$ and $\mathrm{a}_{2}$ associated alleles for the genes located between the two mating-type loci at the time of the linkage event. We built the genealogies of 23, 11 and 8 genes ancestrally located between the PR locus and its centromere, in the PR-chromosome short arm, and between the HD locus and its centromere, respectively. Among these 42 genes, the $31(23+8)$ ancestrally in the PR370 to-centromere and HD-to-centromere regions were initially located between the two matingtype loci for all types of rearrangements documented so far, except in $M$. silenes-acaulis, in which the PR chromosome was fused in the reverse orientation so that the genes ancestrally located between the PR locus and its centromere are still recombining. The genealogies 
recovered in the genes ancestrally located between the PR locus and its centromere and between the HD locus and its centromere displayed trans-specific polymorphism between $M$. lychnidis-dioicae and M. silenes-dioicae on the one hand and M. violaceum s. str. and M. coronariae on the other hand. This indicates that recombination cessation occurred independently in these two clades (Figure 1), either following independent similar chromosomal rearrangements or a single basal chromosomal rearrangement with initially incomplete recombination suppression. We also found trans-specific polymorphism, shared between $M . v$. caroliniana and $M . v$. parryi, in the HD-to-centromere and PR-to-centromere regions, suggesting: 1) an ancestral chromosomal rearrangement and gradual completion of recombination suppression, or 2) recombination suppression between each mating-type locus and its respective centromere, as in M. lagerheimii and $M$. saponariae, and then, indepently 385 in the two species, the same type of chromosomal rearrangement linking the HD and PR loci one two each other. The two steps would be beneficial, as the linkage between each matingtype locus and its respective centromere is beneficial under intra-tetrad selfing, while the HDPR linkage is, in addition, also beneficial under inter-tetrad selfing (Carpentier et al. 2019). Both alternatives are consistent with much older date estimates for recombination 390 suppression in both regions (2.23 or 2.79 MYA) than the inferred speciation date (1.42 MYA, Figure 4 and Supplementary table S5).

The genealogies displayed by the genes ancestrally located in the three regions analysed, i.e. PR-to-centromere, PR short arm and HD-to-centromere, were incongruent for some nodes (Figure 4), in particular for the placement of alternate mating types in the M. v. gracilicaulis -

395 M. v. viscidula - M. v. melanantha clade. We indeed detected trans-specific polymorphism for these three species (i.e., clustering of allele according to mating type rather than according to species) in the gene genealogies of the HD-to-centromere but not the PR-to-centromere ancestral regions. The genes ancestrally located in the PR-chromosome short arm displayed trans-specific polymorphism between $M . v$. viscidula and M. v. melanantha, but not shared 400 with M. v. gracilicaulis, while there was no trans-specific polymorphism at all in this clade in the PR-to-centromere ancestral region. This suggests that full HD-PR linkage in this clade was achieved in several steps, some of which occurred independently in the different species (Figure 4 and Supplementary Figure S5). The chromosomal rearrangement and the suppression of recombination in the HD-to-centromere region have most likely occurred at 405 the base of this three-species clade. Indeed, the ancestral recombination suppression in the HD-to-centromere region would not be beneficial unless the chromosomal rearrangement had 
already occurred, linking the HD and PR loci one to each other. The recombination suppression has then extended after the divergence of $M . v$. gracilicaulis, and then again further after the speciation between M. v. viscidula and M. v. melanantha (Figure 4A, 4B and 4C). This stepwise completion of recombination suppression led to the formation of three distinct evolutionary strata in the $M . v$. viscidula-M. $v$. melanantha clade, that we called light grey, dark grey and black strata (Figure 4E), and which can be also seen in the $\mathrm{d}_{\mathrm{S}}$ plots (Figure 3B). The estimated dates of recombination suppression (Supplementary Table S5) indicated that the light grey stratum (HD-to-centromere) occurred in the M. v. viscidula-M. $v$. melanantha clade 1.5 MYA (in the same event as in the M. v. gracilicaulis lineage), while the dark grey stratum (PR-chromosome short arm) occurred 0.88 MYA and the black strata (PRto-centromere) between 0.57 MYA in M. v. melanantha and 0.6 MYA in M. v. viscidula. In the M. v. gracilicaulis lineage, the black stratum (PR-chromosome short arm plus PR-tocentromere) evolved 0.8 to 0.9 MYA (Supplementary Figure S5).

420 In M. v. lateriflora parasitizing Moehringia lateriflora (Figure 2F) and M. v. parryi parazitizing Silene parryi (Supplementary Figure S4D), the mating-type chromosomes were formed by a fusion between the long arm of the PR chromosome and the short arm of the HD chromosome, following the same scenario as M. v. caroliniana and M. tatarinowii (Figure 1). The placement of $M$. v. parryi as a sister species of $M$. $v$. caroliniana in the phylogeny 425 suggests that the mating-type locus linkage event could have occurred before the divergence of these two species, which was confirmed by the trans-specific polymorphism found between these two species in their black stratum (Figure 4). In contrast, the strongly supported placement of $M$. $v$. lateriflora within the clade formed by $M$. lagerheimii and $M$. saponariae (Figure 1), both with mating-type loci linked to their respective centromere, 430 indicates that the mating-type chromosome rearrangements in $M$. v. lateriflora occurred independently from that in M. v. caroliniana, M. v. parryi and M. v. tatarinowii, representing convergence. The exact same breakpoints were involved in the convergent rearrangements, exactly at the centromere when we consider that the ancestral state of the PR chromosome corresponds to the gene order of M. lagerheimii.

435 We found a large range for the estimated dates of recombination suppression events between mating-type loci, from 0.15 MYA in M. silenes-acaulis to 3.58 MYA M. scabiosae (Figure 4, Supplementary Table S5). In M. scabiosae, the much older dates of recombination suppression in the HD-to-centromere region than in the PR-to-centromere region suggest that, 
in this lineage too, the recombination suppression between the two mating-type loci has likely

\section{5} occurred in two distinct evolutionary strata, which can also be observed in the $\mathrm{d}_{\mathrm{s}}$ plot previously published (Branco et al 20218). Actually, the estimated dates of recombination suppression were more ancient for the HD-to-centromere region than in the long arm of the PR chromosome in most species (Supplementary Table S5); this suggests that recombination was not completely suppressed all along the region between the two mating-type loci immediately after the chromosomal rearrangements in the various species, but extended progressively from the mating-type loci until joining to complete recombination cessation.

\section{Evolutionary strata beyond mating-type loci}

In order to investigate the existence of young evolutionary strata, we inspected the $\mathrm{d}_{\mathrm{s}}$ values between the alleles in $\mathrm{a}_{1}$ and $\mathrm{a}_{2}$ genomes along the mating-type chromosomes according to

450 the ancestral gene order inferred from M. lagerheimii. In all the four species with new genome sequences, $d_{\mathrm{s}}$ was high between alleles at the genes located ancestrally between the mating-type loci, in agreement with the complete recombination suppression linking matingtype loci (Figures 3B, C, D and E). As in previous studies (Branco et al., 2018, 2017), we called the regions without recombination linking the HD and PR loci in each species the black strata; note however that they do not all have the same origin or gene content (Figure 1). In M. v. lateriflora, the moderate level of rearrangements (Figure 2C), together with the low level of differentiation between the genes of the black strata (Figure 3D), confirm that the mating-type locus linkage event occurred very recently (date estimate 0.19 to 0.41 MYA, Supplementary Table S5). More extensive rearrangements between $\mathrm{a}_{1}$ and $\mathrm{a}_{2}$ mating type chromosomes in M. v. gracilicaulis, M. v. viscidula and M. v. parryi (Figure 2C; Supplementary Figures S1A and S1C), as well as higher $d_{S}$ values in their black strata (Figures 3B, C and E), confirm the older linkage of the mating-type loci in these species (Supplementary Table S5).

We found, in all the species studied here, the footprints of the same ancient shared evolutionary strata as in other Microbotryum species, with high $\mathrm{d}_{\mathrm{s}}$ levels in the blue and purple regions in proximity to the HD and PR loci, respectively (Figure 3), thus likely representing stepwise extensions of recombination suppression beyond mating-type loci at the basis of the Microbotryum clade (Figure 1). These different evolutionary strata were not initially delimited only based on their $\mathrm{d}_{\mathrm{s}}$ levels, but by i) the set of species displaying nonzero $d_{s}$ values in these genomic regions and ii) the level of trans-specific polymorphism, two 
strong indicators of the origin of the strata in the phylogeny. Further extension of the recombination suppression beyond the PR locus and its ancient purple stratum was detected in M. v. gracilicaulis and M. v. viscidula (Figures 3B and 3C), corresponding to the previously identified orange stratum in the $M$. lychnidis-dioicae clade as well as in $M$.

475 silenes-acaulis and M. v. paradoxa (Figure 1). In agreement with the previous inference that the orange stratum evolved after the divergence of the $M$. lagerheimii - $M$. saponariae clade (Hartmann et al., 2021), we did not find traces of the orange stratum in M. v. lateriflora or $M$. v. parryi (Figure 3C and 3D). However, the orange stratum seemed to be present in $M . v$. tatarinowii (Fig. 1; Carpentier et al. 2021), which suggests an independent evolution in this

480 later lineage. We did not find on M. v. parryi footprints of the light blue stratum previously identified in M. v. caroliniana (Branco et al. 2018), neither from the $\mathrm{d}_{\mathrm{s}}$ plot or in the transspecific polymorphism analyses, supporting the inference that this stratum evolved very recently in $M . v$. caroliniana, later than the black stratum that is shared with $M$. v. parryi (trans-specific black stratum date 2.23-2.79 MYA, M. v. caroliniana light blue stratum date 0.26 MYA, speciation date 1.42 MYA, Figure 4 and Supplementary Table S5).

\section{Discussion}

In the present study, we obtained high-quality assemblies of alternative mating types for four additional Microbotryum anther-smut fungi, in addition to the 13 already available. We found additional events of independent chromosomal rearrangements bringing the two mating-type

490 loci onto the same chromosome followed by recombination suppression linking the two mating-type loci to each other. In total, this makes at least nine independent and convergent events of mating-type locus linkage across the Microbotryum genus. The dates of recombination suppression between the two mating-type loci ranged from 0.15 to 3.58 million years ago. In addition, we found further strong support for independent events of recombination suppression between mating-type loci and centromeres in two Microbotryum species, through the phylogenetic placement of an additional species whose genomes we sequenced here. With these four events of recombination suppression (two species times two centromere-mating-type locus linkage events), this makes a total of 13 events of recombination suppression involving mating-type loci across the phylogeny of Microbotryum 500 anther-smut fungi, and even more if we count the independent completion events of recombination cessation in the $M . v$. melanantha clade. As the Microbotryum genus likely 
contains more than a hundred species (Hood et al., 2010; Lutz et al., 2008), these results suggest that there remains a rich resource of genomic diversity in the evolution of suppressed recombination in linkage to reproductive compatibility loci. Given the high number of convergent events having linked the two mating-type loci on the same chromosome, one may wonder why it did not occur earlier, at the basis of the Microbotryum clade. A speculative answer is that similar chromosomal rearrangements need to occur on the two mating-type chromosomes to avoid unbalanced meiosis (Fraser et al., 2004), which may not occur that easily, even if the number of convergent events across the phylogeny show that it can happen repeatedly.

Additional independent events of mating-type locus linkage also occurred in other basidiomycete genera, e.g., Ustilago and Cryptococcus (Bakkeren and Kronstad, 1994; Hartmann et al., 2021; Sun et al., 2019). Such striking and repeated convergence shows that strong selection can lead to similar evolution repeatedly. The linkage of HD and PR loci has

515 likely been selected for increasing gamete compatibility odds under selfing (Branco et al 2017, Hood 2002, 2004). With a single locus and two alleles, gametes are indeed compatible with $50 \%$ of other gametes, while the odd is only $25 \%$ with two loci and two alleles each, as fungi can only mate when alleles are different at both loci. Linkage between each of the two mating-type loci and their respective centromere gives the same odds of gamete compatibility

520 as mating-type loci linkage, but only within meiotic tetrads, not among tetrads of a given diploid individual where the odds fall to 25\%. This may be why only two Microbotryum species evolved PR- and HD-centromere linkage without chromosome fusion (Carpentier et al. 2019) whereas HD-PR mating-type loci linkage evolved a dozen times. Such two-fold increase in the odds of compatibility may be important to maximize the chances of plant

525 infection, as a higher number of dikaryons on a given plant increases disease probability (Kaltz et al., 1999; Roche et al., 1995), and it may give a time advantage under competition situations, as a single genotype typically has a resident advantage at parasitizing a plant even when several genotypes are deposited on the plant (Day, 1980; Gibson et al., 2012; Giraud et al., 2005; Hood, 2003; López-Villavicencio et al., 2007). The repeated convergent evolution of mating-type locus linkage shows that natural selection is very powerful in the face of contingency (i.e. the stochasticity associated with initial conditions and mutations occurring randomly) and can make evolution repeatable.

We did not find new types of chromosomal rearrangements having led to HD and PR on a 
single chromosome, and instead found repeated independent evolution of the same four types of chromosome fusion/fission events, with breakpoints at centromeres. This suggests that the convergence events of HD-PR linkage have occurred by convergence of a limited number of chromosomal rearrangement types and that rearrangements at centromeres are more likely than at other places in chromosomes. Such rearrangements at centromeres have also been reported in the other basidiomycetes having undergone HD-PR locus linkage (Sun et al., 540 2017).

In the $M . \quad v$. gracilicaulis-M. $\quad v$. viscidula-M. $\quad v$. melanantha clade, we found that recombination suppression in the HD-to-centromere region was ancestral to the clade while the recombination suppression in the PR short arm was shared only by $M . v$. viscidula and $M$. $v$. melanantha, and the the recombination suppression in the PR-to-centromere region

545 occurred independently in each species, completing the PR-HD linkage. Recombination suppression between the HD locus and its centromere without linkage to PR would bring no advantage in terms of odds of gamete compatibility; the chromosomal rearrangement that has brought the HD and PR loci on the same chromosome is therefore likely ancestral to the clade, which is supported by the trans-specific polymorphism found at some genes in the

550 short arm of the PR chromosome. There would have been initially only partial linkage between HD and PR if recombination was allowed in the PR-to-centromere region, but the increased rates of gamete compatibility would still have been beneficial compared to full independence of mating-type loci. Later, completion of recombination cessation would also have been a beneficial step, further increasing rates of gamete compatibility. We also found evidence for gradual completion of recombination cessation in the M. v. caroliniana - M. $v$. parryi clade.

In conclusion, our findings further support the anther-smut fungi as excellent models to study the evolution of recombination suppression and its consequences, providing many independent cases of recombination cessation across a wide range of ages.

\section{Data access}

Sequencing data and genome assemblies were deposited at GenBank under the BioProject PRJNA771266. 
bioRxiv preprint doi: https://doi.org/10.1101/2021.10.29.466223; this version posted October 30, 2021. The copyright holder for this preprint (which was not certified by peer review) is the author/funder, who has granted bioRxiv a license to display the preprint in perpetuity. It is made available under aCC-BY-NC 4.0 International license.

\section{Acknowledgements}

This work was supported by the National Institute of Health (NIH) grant R15GM119092 to

565 M. E. H., the Louis D. Foundation award and EvolSexChrom ERC advanced grant \#832352

to T. G., and an IDEX Paris-Saclay Bochum University PhD grant to M.D. We thank Kurt Hasselman, John Bain and Hui Tang who collected the strains (with an avoided encounter with a grizzly bear for John Bain). We thank Cécile Fairhead for help with DNA extraction. PacBio sequencing was conducted at the IGM Genomics Center, University of California, 570 San Diego, La Jolla, California

\section{Author contributions}

T.G., R.C.R.d.I.V. and M.E.H designed and supervised the study. T.G., M.E.H, D.B. and M.D. contributed to get funding. F.C., R.C.R.d.I.V. and M.E.H. obtained the genome assemblies. M.D., F.C., R.C.R.d.l.V. performed the genomic analyses. T.G., R.C.R.d.l.V. and 575 M.D. wrote the original draft. All authors contributed to the manuscript.

\section{Conflicts of interest}

The authors have no conflict of interest to declare.

\section{References}

Abascal, F., Zardoya, R., Telford, M.J., 2010. TranslatorX: multiple alignment of nucleotide sequences guided by amino acid translations. Nucleic Acids Res. 38, W7-13. https:// doi.org/10.1093/nar/gkq291

Altschul, S.F., Gish, W., Miller, W., Myers, E.W., Lipman, D.J., 1990. Basic local alignment search tool. J. Mol. Biol. 215, 403-410. https://doi.org/10.1016/S00222836(05)80360-2

585 Bachtrog, D., Mank, J.E., Peichel, C.L., Kirkpatrick, M., Otto, S.P., Ashman, T.-L., Hahn, M.W., Kitano, J., Mayrose, I., Ming, R., Perrin, N., Ross, L., Valenzuela, N., Vamosi, J.C., 2014. Sex determination: why so many ways of doing it? PLoS Biol. 12, e1001899. https://doi.org/10.1371/journal.pbio.1001899

Badouin, H., Hood, M.E., Gouzy, J., Aguileta, G., Siguenza, S., Perlin, M.H., Cuomo, C.A., Fairhead, C., Branca, A., Giraud, T., 2015. Chaos of Rearrangements in the MatingType Chromosomes of the Anther-Smut Fungus Microbotryum lychnidis-dioicae. 
Bakkeren, G., Kronstad, J.W., 1994. Linkage of mating-type loci distinguishes bipolar from tetrapolar mating in basidiomycetous smut fungi. Proc. Natl. Acad. Sci. 91, 70857089. https://doi.org/10.1073/pnas.91.15.7085

Bao, Z., Eddy, S.R., 2002. Automated de novo identification of repeat sequence families in sequenced genomes. Genome Res. 12, 1269-1276. https://doi.org/10.1101/gr.88502

Bazzicalupo, A.L., Carpentier, F., Otto, S.P., Giraud, T., 2019. Little Evidence of Antagonistic Selection in the Evolutionary Strata of Fungal Mating-Type Chromosomes (Microbotryum lychnidis-dioicae). G3 GenesGenomesGenetics 9, 1987-1998. https:// doi.org/10.1534/g3.119.400242

Benson, G., 1999. Tandem repeats finder: a program to analyze DNA sequences. Nucleic Acids Res. 27, 573-580. https://doi.org/10.1093/nar/27.2.573

Bergero, R., Charlesworth, D., 2009. The evolution of restricted recombination in sex chromosomes. Trends Ecol. Evol. 24, 94-102. https://doi.org/10.1016/j.tree.2008.09.010

Bouckaert, R., Heled, J., Kühnert, D., Vaughan, T., Wu, C.-H., Xie, D., Suchard, M.A., Rambaut, A., Drummond, A.J., 2014. BEAST 2: A software platform for bayesian evolutionary analysis. PLoS Comput. Biol. 10, e1003537. https://doi.org/10.1371/journal.pcbi.1003537

Branco, S., Badouin, H., Rodríguez de la Vega, R.C., Gouzy, J., Carpentier, F., Aguileta, G., Siguenza, S., Brandenburg, J.-T., Coelho, M.A., Hood, M.E., Giraud, T., 2017. Evolutionary strata on young mating-type chromosomes despite the lack of sexual antagonism. Proc. Natl. Acad. Sci. U. S. A. 114, 7067-7072. https://doi.org/10.1073/pnas.1701658114

Branco, S., Carpentier, F., Rodríguez de la Vega, R.C., Badouin, H., Snirc, A., Le Prieur, S., Coelho, M.A., de Vienne, D.M., Hartmann, F.E., Begerow, D., Hood, M.E., Giraud, T., 2018. Multiple convergent supergene evolution events in mating-type chromosomes. Nat. Commun. 9. https://doi.org/10.1038/s41467-018-04380-9

Carpentier, F., Rodríguez de la Vega, R.C., Branco, S., Snirc, A., Coelho, M.A., Hood, M.E., Giraud, T., 2019. Convergent recombination cessation between mating-type genes and centromeres in selfing anther-smut fungi. Genome Res. 29, 944-953. https://doi.org/10.1101/gr.242578.118

Carpentier, F., Rodríguez de la Vega, R.C., Perlin, M.H., Wallen, R.M., Hood, M.E., Giraud, T., 2021. Tempo of degeneration across independently evolved non-recombining regions 2021.07.20.453045. https://doi.org/10.1101/2021.07.20.453045

Chaisson, M.J., Tesler, G., 2012. Mapping single molecule sequencing reads using basic local alignment with successive refinement (BLASR): application and theory. BMC Bioinformatics 13, 238. https://doi.org/10.1186/1471-2105-13-238

Charlesworth, D., 2017. Evolution of recombination rates between sex chromosomes. Philos. Trans. R. Soc. B Biol. Sci. 372, 20160456. https://doi.org/10.1098/rstb.2016.0456

Chin, C.-S., Alexander, D.H., Marks, P., Klammer, A.A., Drake, J., Heiner, C., Clum, A., Copeland, A., Huddleston, J., Eichler, E.E., Turner, S.W., Korlach, J., 2013. Nonhybrid, finished microbial genome assemblies from long-read SMRT sequencing data. Nat. Methods 10, 563-569. https://doi.org/10.1038/nmeth.2474

Coelho, M.A., Bakkeren, G., Sun, S., Hood, M.E., Giraud, T., 2017. Fungal sex: The basidiomycota. Microbiol. Spectr. 5. https://doi.org/10.1128/microbiolspec.FUNK0046-2016

Consortium TU, 2011. Ongoing and future developments at the Universal Protein Resource. Nucleic Acids Res. 39, D214-D219. https://doi.org/10.1093/nar/gkq1020

Day, A.W., 1980. Competition and distribution studies of genetically marked strains of Ustilago violacea in the same host plant. Bot. Gaz. 141, 313-320. https://doi.org/10.1086/337162

Devier, B., Aguileta, G., Hood, M.E., Giraud, T., 2009. Ancient trans-specific polymorphism at pheromone receptor genes in Basidiomycetes. Genetics 181, 209-223. 
Edgar, R.C., 2004. MUSCLE: a multiple sequence alignment method with reduced time and space complexity. BMC Bioinformatics 5, 113. https://doi.org/10.1186/1471-2105-5113

Ekseth, O.K., Kuiper, M., Mironov, V., 2014. orthAgogue: an agile tool for the rapid prediction of orthology relations. Bioinformatics 30, 734-736. https://doi.org/10.1093/bioinformatics/btt582

Ellinghaus, D., Kurtz, S., Willhoeft, U., 2008. LTRharvest, an efficient and flexible software for de novo detection of LTR retrotransposons. BMC Bioinformatics 9, 18. https://doi.org/10.1186/1471-2105-9-18

Foissac, S., Gouzy, J., Rombauts, S., Mathe, C., Amselem, J., Sterck, L., de Peer, Y., Rouze, P., Schiex, T., 2008. Genome Annotation in Plants and Fungi: EuGene as a Model Platform. Curr. Bioinforma. 3, 87-97. https://doi.org/10.2174/157489308784340702

Foulongne-Oriol, M., Taskent, O., Kües, U., Sonnenberg, A.S.M., van Peer, A.F., Giraud, T., 2021. Mating-type locus organization and mating-type chromosome differentiation in the bipolar edible button mushroom Agaricus bisporus. Genes 12, 1079. https://doi.org/10.3390/genes12071079

Fraser, J.A., Diezmann, S., Subaran, R.L., Allen, A., Lengeler, K.B., Dietrich, F.S., Heitman, J., 2004. Convergent evolution of chromosomal sex-determining regions in the animal and fungal kingdoms. PLoS Biol. 2, e384. https://doi.org/10.1371/journal.pbio.0020384

Gibson, A.K., Hood, M.E., Giraud, T., 2012. Sibling competition arena: selfing and a competition arena can combine to constitute a barrier to gene flow in sympatry. Evol. Int. J. Org. Evol. 66, 1917-1930. https://doi.org/10.1111/j.1558-5646.2011.01563.x

Giraud, T., Jonot, O., Shykoff, J.A., 2005. Selfing propensity under choice conditions in a parasitic fungus, Microbotryum violaceum, and parameters influencing infection success in artificial ioculations. Int. J. Plant Sci. 166, 649-657. https://doi.org/10.1086/430098

Guindon, S., Dufayard, J.-F., Lefort, V., Anisimova, M., Hordijk, W., Gascuel, O., 2010. New algorithms and methods to estimate maximum-likelihood phylogenies: assessing the performance of PhyML 3.0. Syst. Biol. 59, 307-321. https://doi.org/10.1093/sysbio/syq010

Hartmann, F.E., Duhamel, M., Carpentier, F., Hood, M.E., Foulongne $\square$ Oriol, M., Silar, P., Malagnac, F., Grognet, P., Giraud, T., 2021a. Recombination suppression and evolutionary strata around mating $\square$ type loci in fungi: documenting patterns and understanding evolutionary and mechanistic causes. New Phytol. 229, 2470-2491. https://doi.org/10.1111/nph.17039

Hartmann, F.E., Rodríguez de la Vega, R.C., Carpentier, F., Gladieux, P., Cornille, A., Hood, M.E., Giraud, T., 2019. Understanding adaptation, coevolution, host specialization, and mating system in castrating anther-smut fungi by combining population and comparative genomics. Annu. Rev. Phytopathol. 57, 431-457. https://doi.org/10.1146/annurev-phyto-082718-095947

Hoang, D.T., Chernomor, O., von Haeseler, A., Minh, B.Q., Vinh, L.S., 2018. UFBoot2: Improving the ultrafast bootstrap approximation. Mol. Biol. Evol. 35, 518-522. https:// doi.org/10.1093/molbev/msx281

Hood, M.E., 2003. Dynamics of multiple infection and within-host competition by the anthersmut pathogen. Am. Nat. 162, 122-133. https://doi.org/10.1086/375539

Hood, M.E., Antonovics, J., Koskella, B., 2004. Shared Forces of Sex Chromosome Evolution in Haploid-Mating and Diploid-Mating Organisms. Genetics 168, 141-146. https://doi.org/10.1534/genetics.104.029900

Hood, M.E., Mena-Alí, J.I., Gibson, A.K., Oxelman, B., Giraud, T., Yockteng, R., Arroyo, M.T.K., Conti, F., Pedersen, A.B., Gladieux, P., Antonovics, J., 2010. Distribution of the anther-smut pathogen Microbotryum on species of the Caryophyllaceae. New Phytol. 187, 217-229. https://doi.org/10.1111/j.1469-8137.2010.03268.x 
700

705

710

715

Ironside, J.E., 2010. No amicable divorce? Challenging the notion that sexual antagonism drives sex chromosome evolution. BioEssays 32, 718-726. https://doi.org/10.1002/bies.200900124

Jay, P., Tezenas, E., Giraud, T., 2021. A deleterious mutation-sheltering theory for the evolution of sex chromosomes and supergenes. bioRxiv 2021.05.17.444504. https:// doi.org/10.1101/2021.05.17.444504

Kaltz, O., Gandon, S., Michalakis, Y., Shykoff, J.A., 1999. Local maladaptation in the anthersmut fungus Microbotryum violaceum to its host plant Silene latifolia: evidence from a cross-inoculation experiment. Evol. Int. J. Org. Evol. 53, 395-407. https://doi.org/10.1111/j.1558-5646.1999.tb03775.x

Kalyaanamoorthy, S., Minh, B.Q., Wong, T.K.F., von Haeseler, A., Jermiin, L.S., 2017. ModelFinder: fast model selection for accurate phylogenetic estimates. Nat. Methods 14, 587-589. https://doi.org/10.1038/nmeth.4285

Kishino, H., Miyata, T., Hasegawa, M., 1990. Maximum likelihood inference of protein phylogeny and the origin of chloroplasts. J. Mol. Evol. 31, 151-160. https://doi.org/10.1007/BF02109483

Koren, S., Walenz, B.P., Berlin, K., Miller, J.R., Bergman, N.H., Phillippy, A.M., 2017. Canu: scalable and accurate long-read assembly via adaptive k-mer weighting and repeat separation. Genome Res. 27, 722-736. https://doi.org/10.1101/gr.215087.116

Lahn, B.T., Page, D.C., 1999. Four evolutionary strata on the human X chromosome. Science 286, 964-967. https://doi.org/10.1126/science.286.5441.964

Le Gac, M., Hood, M.E., Fournier, E., Giraud, T., 2007a. Phylogenetic evidence of hostspecific cryptic species in the anther smut fungus. Evolution 61, 15-26. https://doi.org/10.1111/j.1558-5646.2007.00002.x

Le Gac, M., Hood, M.E., Giraud, T., 2007b. Evolution of reproductive isolation within a parasitic fungal species complex. Evol. Int. J. Org. Evol. 61, 1781-1787. https://doi.org/10.1111/j.1558-5646.2007.00144.x

Li, H., Handsaker, B., Wysoker, A., Fennell, T., Ruan, J., Homer, N., Marth, G., Abecasis, G., Durbin, R., 2009. The Sequence Alignment/Map format and SAMtools. Bioinformatics 25, 2078-2079. https://doi.org/10.1093/bioinformatics/btp352

López-Villavicencio, M., Jonot, O., Coantic, A., Hood, M.E., Enjalbert, J., Giraud, T., 2007. Multiple infections by the anther smut pathogen are frequent and involve related strains. PLOS Pathog. 3, e176. https://doi.org/10.1371/journal.ppat.0030176

Lutz, M., Piątek, M., Kemler, M., Chlebicki, A., Oberwinkler, F., 2008. Anther smuts of Caryophyllaceae: Molecular analyses reveal further new species. Mycol. Res. 112, 1280-1296. https://doi.org/10.1016/j.mycres.2008.04.010

Menkis, A., Jacobson, D.J., Gustafsson, T., Johannesson, H., 2008. The Mating-Type Chromosome in the Filamentous Ascomycete Neurospora tetrasperma Represents a Model for Early Evolution of Sex Chromosomes. PLoS Genet. 4. https://doi.org/10.1371/journal.pgen.1000030

Minh, B.Q., Nguyen, M.A.T., von Haeseler, A., 2013. Ultrafast approximation for phylogenetic bootstrap. Mol. Biol. Evol. 30, 1188-1195. https://doi.org/10.1093/molbev/mst024

Minh, B.Q., Schmidt, H.A., Chernomor, O., Schrempf, D., Woodhams, M.D., von Haeseler, A., Lanfear, R., 2020. IQ-TREE 2: New models and efficient methods for phylogenetic inference in the genomic era. Mol. Biol. Evol. 37, 1530-1534. https://doi.org/10.1093/molbev/msaa015

Petit, E., Giraud, T., de Vienne, D.M., Coelho, M.A., Aguileta, G., Amselem, J., Kreplak, J., Poulain, J., Gavory, F., Wincker, P., Young, S.K., Cuomo, C., Perlin, M.H., Hood, M.E., 2012. Linkage to the mating-type locus across the genus Microbotryum: insights into non-recombining chromosomes. Evol. Int. J. Org. Evol. 66, 3519-3533. https://doi.org/10.1111/j.1558-5646.2012.01703.x

Price, A.L., Jones, N.C., Pevzner, P.A., 2005. De novo identification of repeat families in large genomes. Bioinforma. Oxf. Engl. 21 Suppl 1, i351-358.

https://doi.org/10.1093/bioinformatics/bti1018 
755 Ranwez, V., Douzery, E.J.P., Cambon, C., Chantret, N., Delsuc, F., 2018. MACSE v2: Toolkit for the alignment of coding sequences accounting for frameshifts and stop codons. Mol. Biol. Evol. 35, 2582-2584. https://doi.org/10.1093/molbev/msy159

Roche, B.M., Alexander, H.M., Maltby, A.D., 1995. Dispersal and disease gradients of anther-smut infection of Silene alba at different life stages. Ecology 76, 1863-1871. https://doi.org/10.2307/1940719

Shimodaira, H., 2002. An approximately unbiased test of phylogenetic tree selection. Syst. Biol. 51, 492-508. https://doi.org/10.1080/10635150290069913

Smit, A.F.A., Hubley, R., 2008. RepeatModeler Open-1.0. URL http://www.repeatmasker.org

Sun, S., Coelho, M.A., Heitman, J., Nowrousian, M., 2019. Convergent evolution of linked mating-type loci in basidiomycete fungi. PLOS Genet. 15, e1008365. https://doi.org/10.1371/journal.pgen.1008365

Sun, S., Yadav, V., Billmyre, R.B., Cuomo, C.A., Nowrousian, M., Wang, L., Souciet, J.-L., Boekhout, T., Porcel, B., Wincker, P., Granek, J.A., Sanyal, K., Heitman, J., 2017. Fungal genome and mating system transitions facilitated by chromosomal translocations involving intercentromeric recombination. PLoS Biol. 15, e2002527. https://doi.org/10.1371/journal.pbio.2002527

van Dongen, S., 2000. Graph clustering by flow simulation. PhD Thesis Cent. Math Comput. Sci. CWI.

Wicker, T., Sabot, F., Hua-Van, A., Bennetzen, J.L., Capy, P., Chalhoub, B., Flavell, A., Leroy, P., Morgante, M., Panaud, O., Paux, E., SanMiguel, P., Schulman, A.H., 2007. A unified classification system for eukaryotic transposable elements. Nat. Rev. Genet. 8, 973-982. https://doi.org/10.1038/nrg2165

Wickham, H., 2009. ggplot2: Elegant graphics for data analysis, Use R! Springer-Verlag, New York. https://doi.org/10.1007/978-0-387-98141-3

780 Yang, Z., 2007. PAML 4: Phylogenetic analysis by maximum likelihood. Mol. Biol. Evol. 24, 1586-1591. https://doi.org/10.1093/molbev/msm088 


\section{Figures}

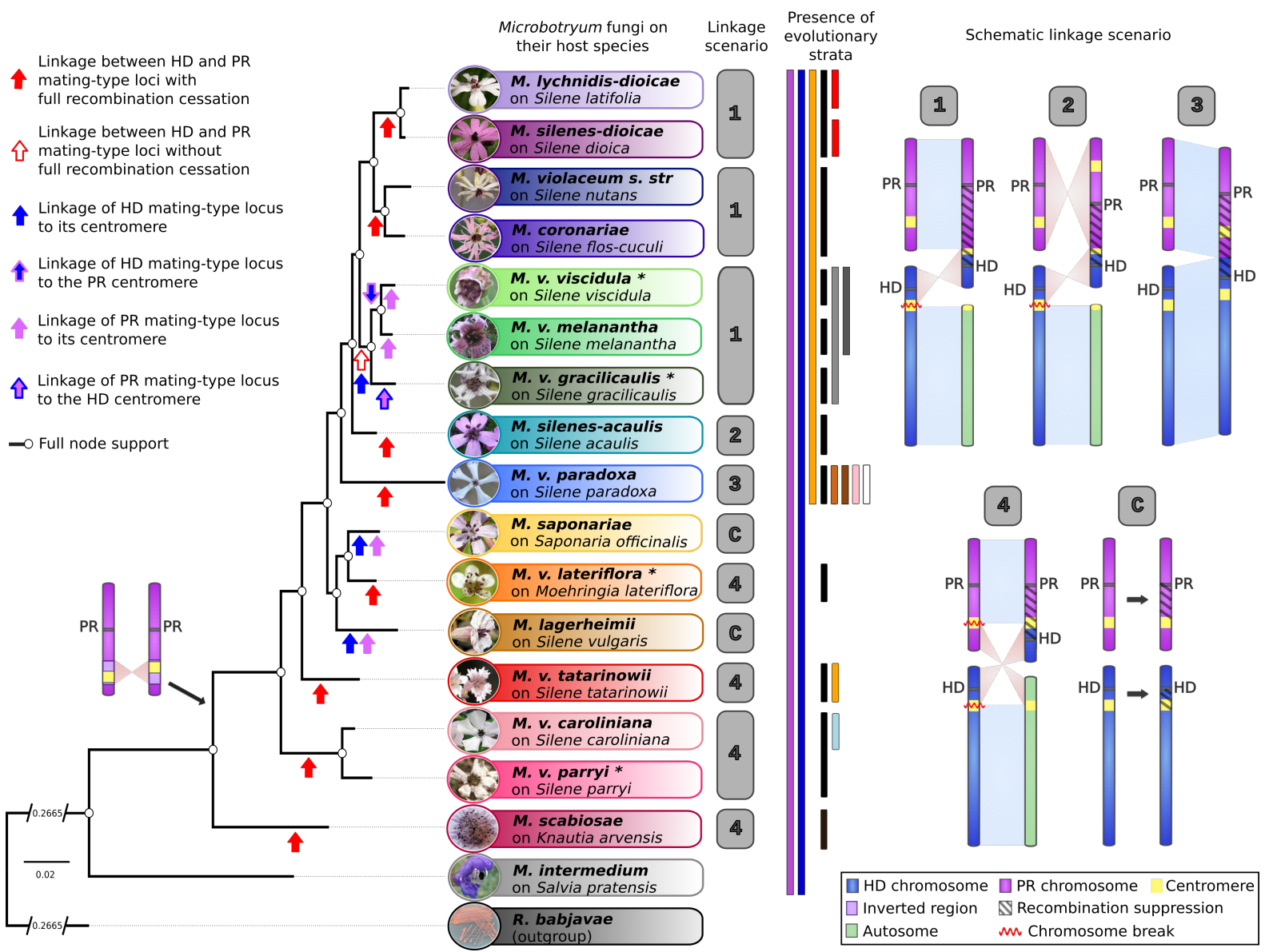

Figure 1: Multiple independent events of recombination suppression in mating-type chromosomes across the Microbotryum phylogeny. Phylogeny of 17 Microbotryum species

rooted by a red yeast outgroup (Rhodothorula babjavae), based on single-copy orthologous gene genealogies (left panel), with pictures of diseased plants. White circles at nodes indicate full support by bootstrap and Shimodaira-Hasegawa-approximate likelihood ratio tests (SHaLRT). The genomes of the species labelled with * were generated for this study. Photos of the diseased flowers by M. E. Hood, except M. v. viscidula and M. v. gracilicaulis (by H.

790 Tang) and M. v. parryi (by acorn13 @iNaturalist, cropped). CC BY 4.0. The red arrows represent the independent events of pheromone-receptor (PR) and homeodomain (HD) mating-type locus linkage. The blue and purple arrows represent the independent events of HD and PR mating-type locus linkage to their respective centromeres, respectively. The purple-framed blue arrow represents the event of HD mating-type locus linkage to the ancestral PR centromere and the blue-framed purple arrow represents the events of PR mating-type locus linkage to the ancestral HD centromere. The chromosomal fission and fusion scenarios having led to HD-PR linkage are indicated right to the species tree (labeled 
with numbers, in grey squares corresponding to independent events). The scenario labeled "C" corresponds to recombination suppression events of mating-type loci to their respective

800 centromeres and is depicted on the right panel. The color bars correspond to the presence of the different evolutionary strata in each species. On the depicted chromosomes are represented in purple the ancestral or fused part of the PR chromosome, in blue the ancestral or fused part of the HD chromosome, in green the regions of the ancestral PR and HD chromosomes that became autosomal following HD-PR linkage. The inversion having 805 occurred near the centromere of the PR chromosome is represented in light purple. The centromeres are represented in yellow. Non-recombining regions (NRRs) are cross-hatched. Chromosome fissions are indicated by red waves. 

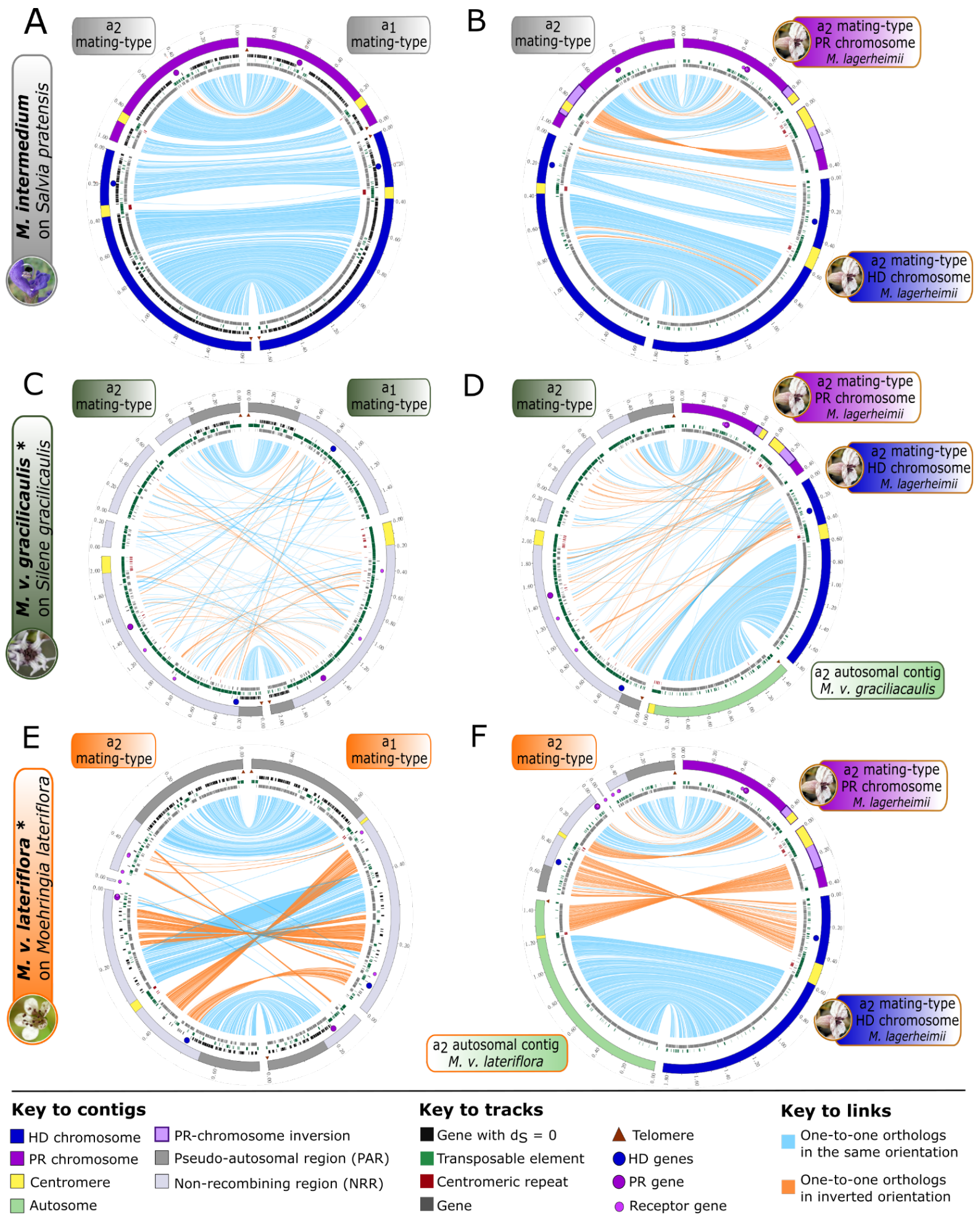

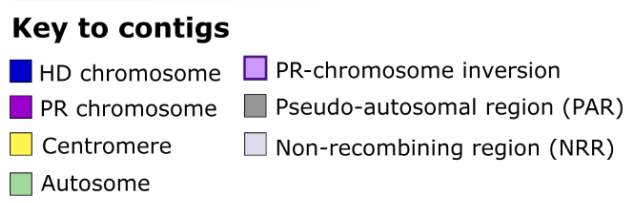

Figure 2: Inter- and intra-specific comparison of gene order between mating-type chromosomes in Microbotryum fungi. Synteny plots between A) homeodomain (HD) and

810 B) pheromone-receptor (PR) chromosomes of opposite mating types in M. lagerheimii, C) $\mathrm{a}_{1}$ and $\mathrm{a}_{2}$ mating-type chromosomes of $M . v$ gracilicaulis, D) $\mathrm{a}_{2}$ mating-type chromosome of $M$. lagerheimii and their homologues in $M . v$. gracilicaulis, E) $\mathrm{a}_{1}$ and $\mathrm{a}_{2}$ mating-type chromosomes of M. v. lateriflora, F) a $\mathrm{a}_{2}$ mating-type chromosomes in M. lagerheimii and their 
homologues in M. v. lateriflora. Comparisons of M. lagerheimii $\mathrm{a}_{2} \mathrm{HD}$ and PR chromosomes and M. v. gracilicaulis (D) and M. v. lateriflora (F) $\mathrm{a}_{2}$ mating-type contigs to infer chromosomal rearrangements having led to HD-PR linkage, considering M. lagerheimii chromosomes as a proxy for the ancestral chromosomal state. The PR, HD and pheromone genes are represented by purple, blue and small light purple circles, respectively. The outer track represents contigs, with length ticks every $200 \mathrm{~kb}$. The HD and PR mating-type 820 chromosomes of $M$. lagerheimii are represented in blue and purple, respectively (A, B, D, F). The region of the inversion having occurred after divergence with M. scabiosae (Fig. 1), encompassing the centromere of the PR chromosome, is highlighted in light purple. For $M . v$. gracilicaulis (C,D) and M. v. lateriflora (E,F), the non-recombining region (NRR) and the pseudo-autosomal region (PAR) of the mating-type chromosomes are represented in light and

825 dark gray, respectively. Green contigs correspond to autosomal contigs of M. v. gracilicaulis (D) and M. v. lateriflora (F). The centromeres are represented in yellow. The telomeres are indicated by brown triangles. Black marks on the inner track represent the genes with null synonymous divergence between $\mathrm{a}_{1}$ and $\mathrm{a}_{2}$ alleles. Green marks on the inner track represent transposable element (TE) copies. Gray marks on the inner track correspond to genes. Red marks on the inner track correspond to the putative centromeric repeats. Blue and orange lines link alleles, the latter corresponding to inversions. The link width is proportional to the corresponding gene length. 

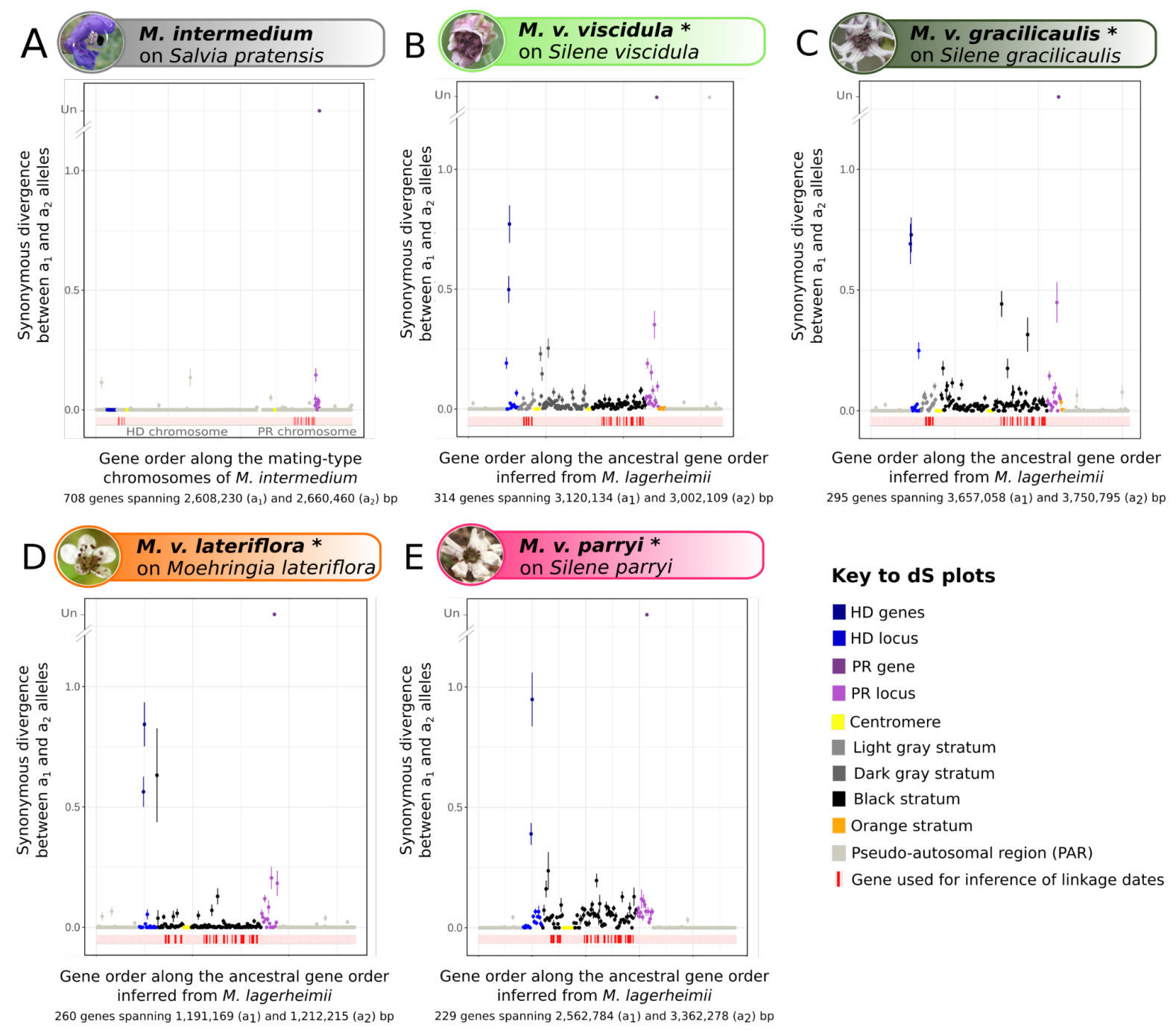

Figure 3: Evolutionary strata in the mating-type chromosomes of Microbotryum fungi.

Per-gene synonymous divergence between $\mathrm{a}_{1}$ and $\mathrm{a}_{2}$ alleles on the mating-type chromosomes of A) M. intermedium, B) M. v. viscidula, C) M. v. gracilicaulis, D) M. v. lateriflora and E) M. v. parryi, plotted along the ancestral gene order, inferred from M. lagerheimii with unlinked mating-type loci. The number of genes and average of the $\mathrm{a}_{1}$ and $\mathrm{a}_{2}$ contig lengths are indicated on the $\mathrm{X}$ axis. Synonymous divergence is used as a proxy for time since recombination suppression. Ancient purple and blue evolutionary strata were formed around each of the mating-type genes (pheromone-receptor, PR, in dark purple, and homeodomain, $\mathrm{HD}$, in dark blue, genes controlling mating compatibility) and is ancestral in the Microbotryum clade (present in all species, including $M$. intermedium in A). In $M . v$. viscidula (B) recombination suppression first linked the HD locus to its centromere, then extended to the PR centromere and eventually to the PR locus, generating the light gray, dark gray and the black strata. In M. v. gracilicaulis (C), recombination suppression linked the HD locus to its centromere and then to the PR locus, forming the light gray and the black strata. 
In M. v. lateriflora (D) and M. v. parryi (E), recombination suppression has linked the HD and PR mating-type loci together, generating black strata. Further extension of recombination suppression beyond the mating-type loci generated the younger orange evolutionary stratum

850 in M. v. viscidula (B) and M. v. gracilicaulis (C). The recombining regions of the mating-type chromosomes with null synonymous divergence, called pseudo-autosomal regions (PARs), are represented in gray. Red ticks below the $\mathrm{X}$ axis correspond to genes used to infer the linkage dates. 
A

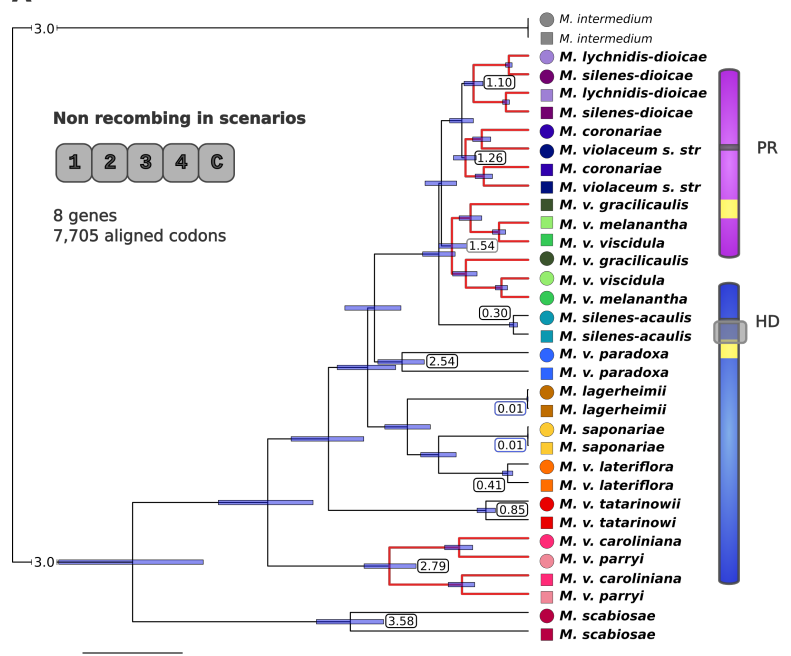

B

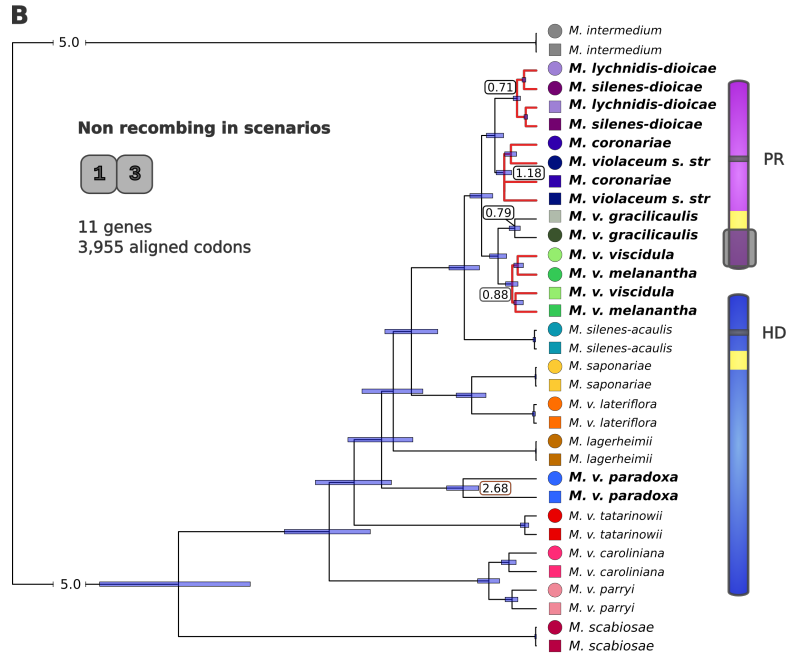

C

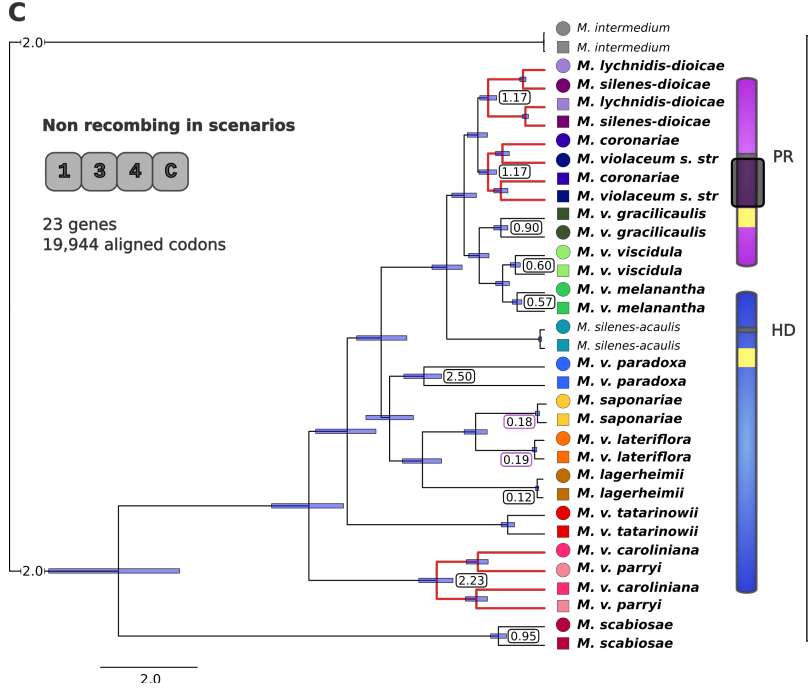

D

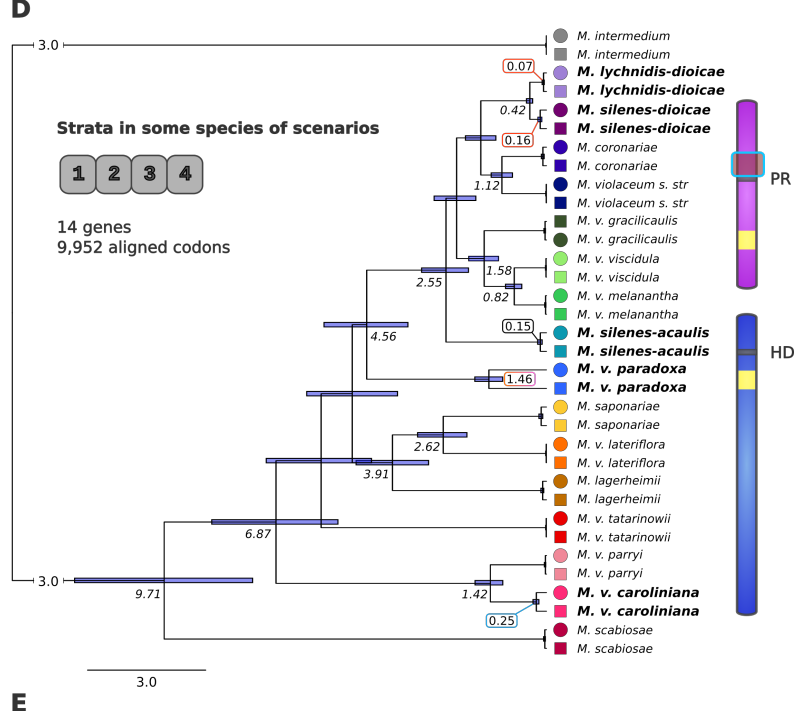

E

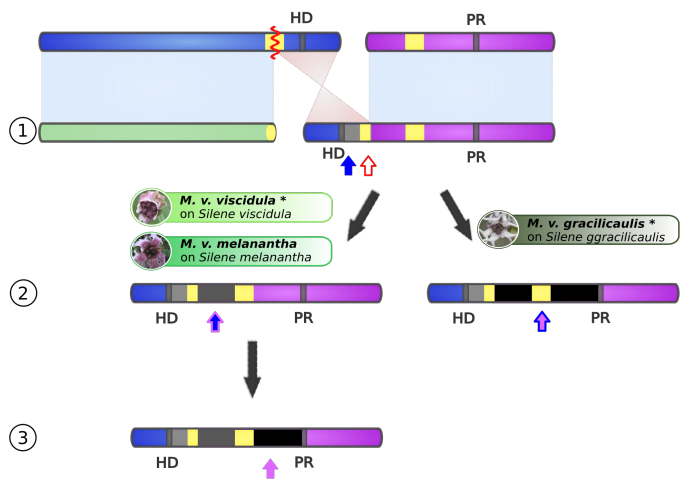

Figure 4: Onset of suppression of recombination date estimates and reconstructed scenario of stepwise recombination suppression between the $\mathrm{HD}$ and PR mating-type loci in the Microbotryum violaceum melanantha clade. A-D) Timetree of conserved genes in the regions boxed on the right-side ancestral chromosome sketches. Topologies are all 
significantly different (AU test p-value > 0.05). Trans-specific polymorphisms are indicated by red branches. Date estimates are shown in colored boxes near the split between $\mathrm{a}_{1}$ and $\mathrm{a}_{2}$

860 alleles, confidence intervals (CI) depicted as rectangles at corresponding nodes. Numbers in italics below branching points in $\mathrm{D}$ correspond to speciation dates. Topology in $\mathrm{D}$ is identical to the species phylogeny and estimated speciation dates overlap. Insets detail the scenarios in which the conserved genes are in non-recombining regions (species names in bold), the number of genes concatenated and the alignment length in codons. See Figure 1 for diagrams

865 of the reconstructed scenarios. Colored symbols correspond to $\mathrm{a}_{1}$ (circles) and $\mathrm{a}_{2}$ alleles (squares) following the species color scheme in Figure 1. E) Reconstructed stepwise recombination suppression having generated the light gray, dark grey and black evolutionary strata in the Microbotryum violaceum melanantha clade. Arrows correspond to the suppression of recombination steps. See supplementary Figure S5 for details on the steps.

870 Box: Key to symbols in the figure. 

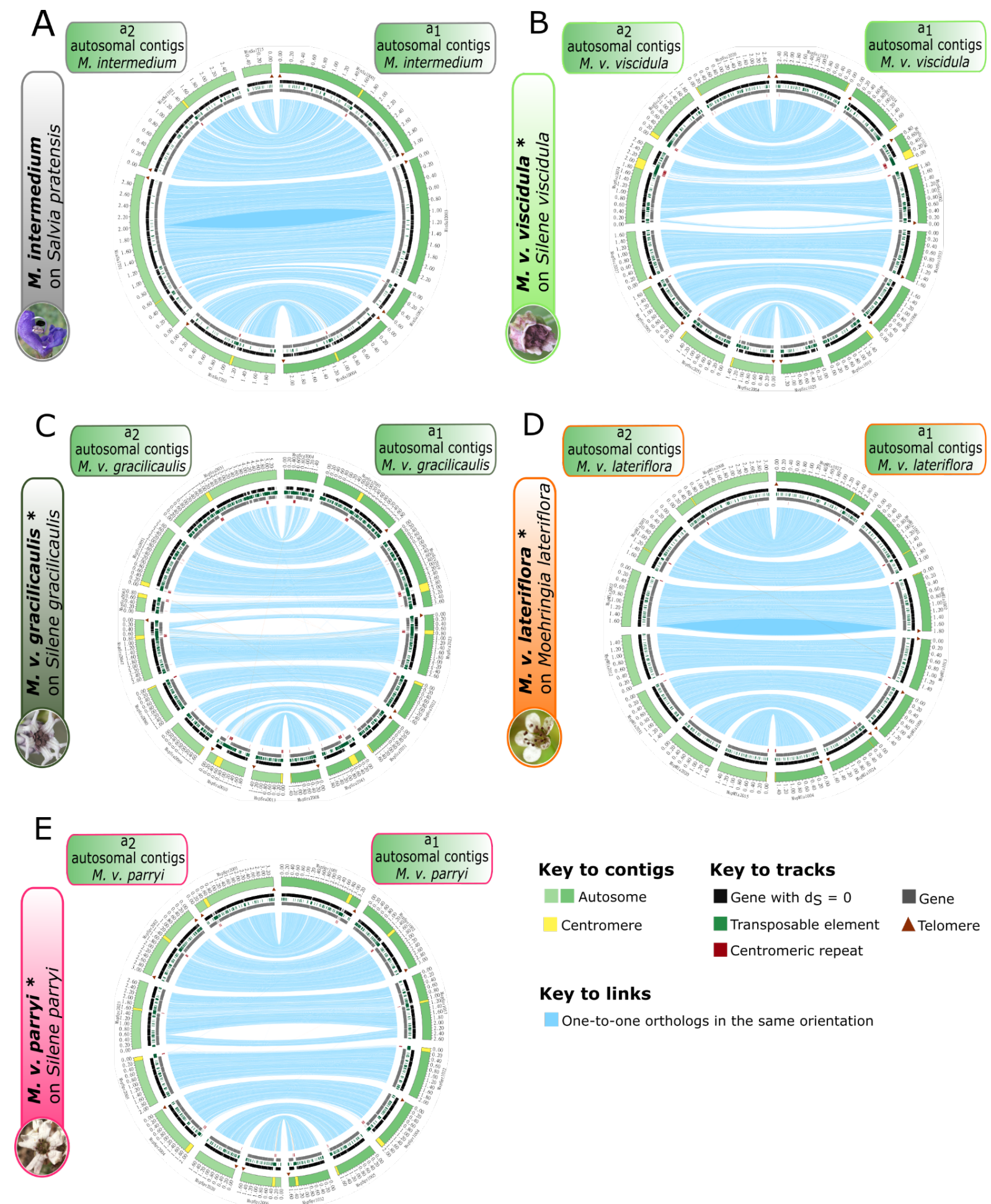

\section{Key to links}

One-to-one orthologs in the same orientation

Supplementary figure S1: Comparison of gene order between haploid genomes of opposite mating types for the largest autosomal contigs in Microbotryum intermedium,

M. violaceum viscidula, M. v. gracilicaulis, M. v. lateriflora and M. v. parryi. Comparison of gene orders between $\mathrm{a}_{1}$ and $\mathrm{a}_{2}$ haploid genomes for autosomal contigs in A) $M$.

875 intermedium, B) M. v. viscidula, C) M. v. gracilicaulis, D) M. v. lateriflora and D) M. v. parryi. The outer track represents contigs, with length ticks every $200 \mathrm{~kb}$. Contigs of the haploid genomes of $\mathrm{a}_{1}$ and $\mathrm{a}_{2}$ mating types are depicted in light green and green, respectively. 
The centromeres are represented in yellow. The telomeres are indicated by brown triangles. Black marks on the inner track represent the genes with null synonymous divergence between $\mathrm{a}_{1}$ and $\mathrm{a}_{2}$ alleles. Green marks on the inner track represent transposable element (TE) copies. Gray marks on the inner track correspond to genes. Red marks on the inner track correspond to the putative centromeric repeats. The link width is proportional to the corresponding gene length. Blue links link alleles in the same orientation.
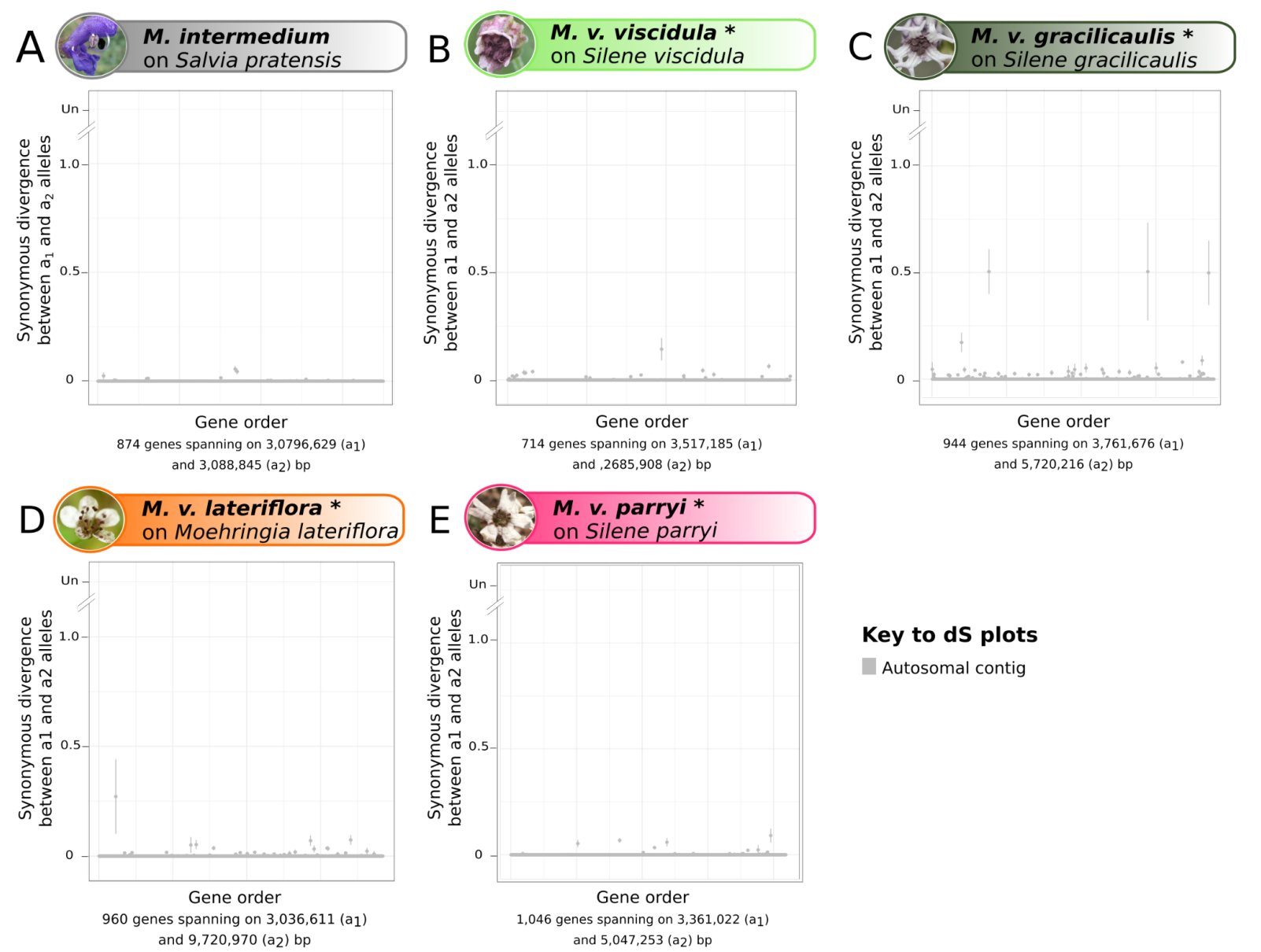

enes spanning on $3,761,676$ (a)

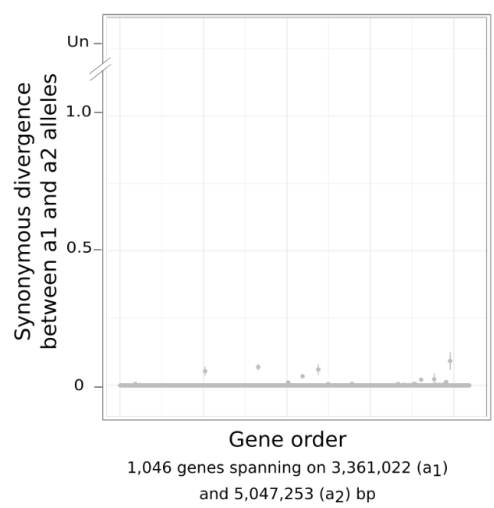

\section{Key to dS plots}

Autosomal contig

Supplementary figure S2: Differentiation between alleles of haploid genomes isolated

885 from a diploid individual, along autosomal contigs in Microbotryum fungi. Per-gene synonymous divergence between alleles present in the haploid $\mathrm{a}_{1}$ and $\mathrm{a}_{2}$ genomes from $\mathrm{a}$ given diploid individual, along the largest autosomal contigs of each of A) M. intermedium, B) M. v. viscidula, C) M. v. gracilicaulis, D) M. v. lateriflora and E) M. v. parryi, plotted along the chromosome. The number of genes and average of the $\mathrm{a}_{1}$ and $\mathrm{a}_{2}$ contig lengths are indicated on the $\mathrm{X}$ axis. 
bioRxiv preprint doi: https://doi org/10.1101/2021 10.29.466223. this version posted October 30, 2021. The copyright holder for this preprint (which was not certified by peer review) is the author/funder, who has granted bioRxiv a license to display the preprint in perpetuity. It is made available under aCC-BY-NC 4.0 International license.
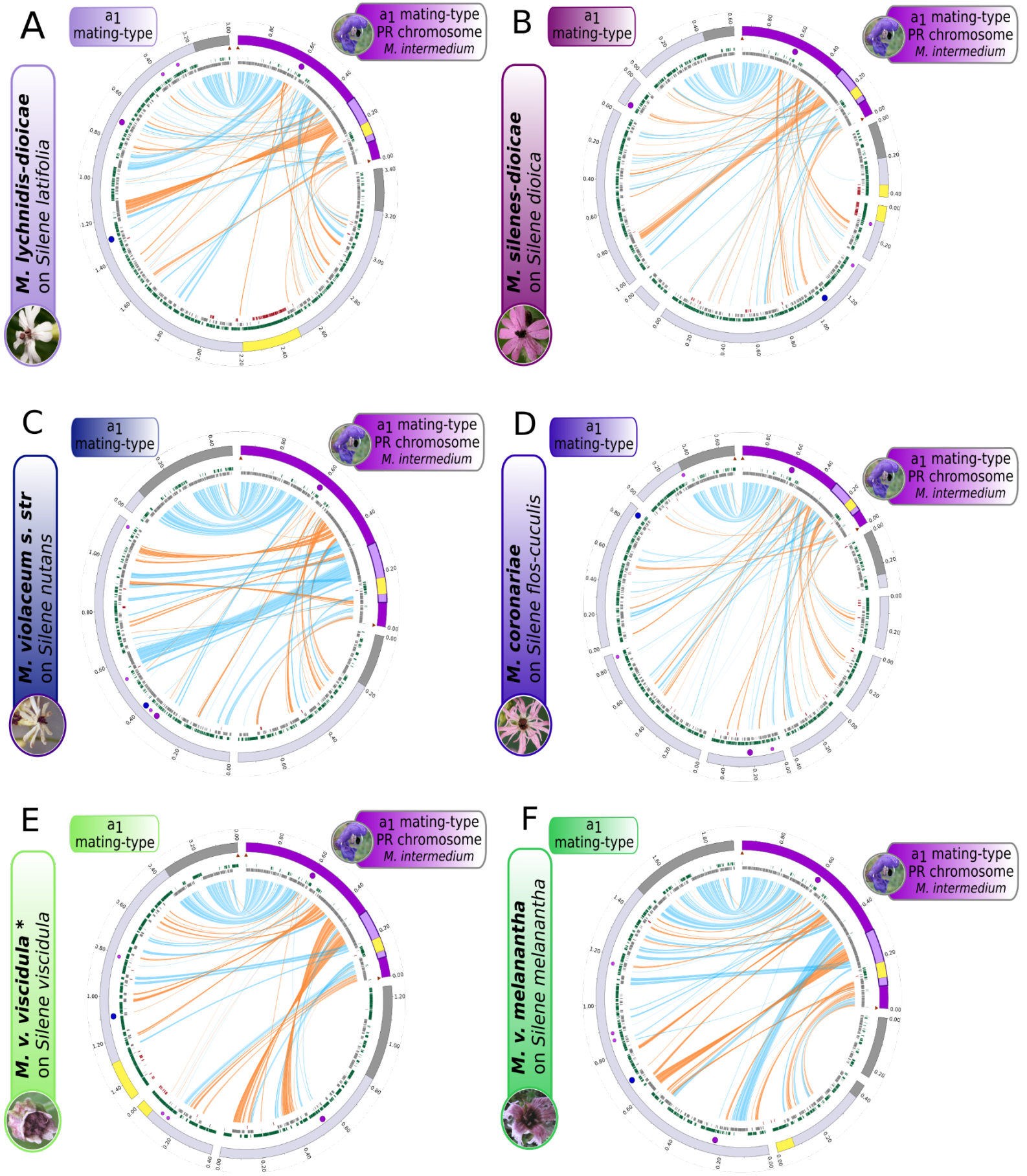
bioRxiv preprint doi: https://doi org/10.1101/2021 10.29.466223. this version posted October 30, 2021. The copyright holder for this preprint (which was not certified by peer review) is the author/funder, who has granted bioRxiv a license to display the preprint in perpetuity. It is made available under aCC-BY-NC 4.0 International license.
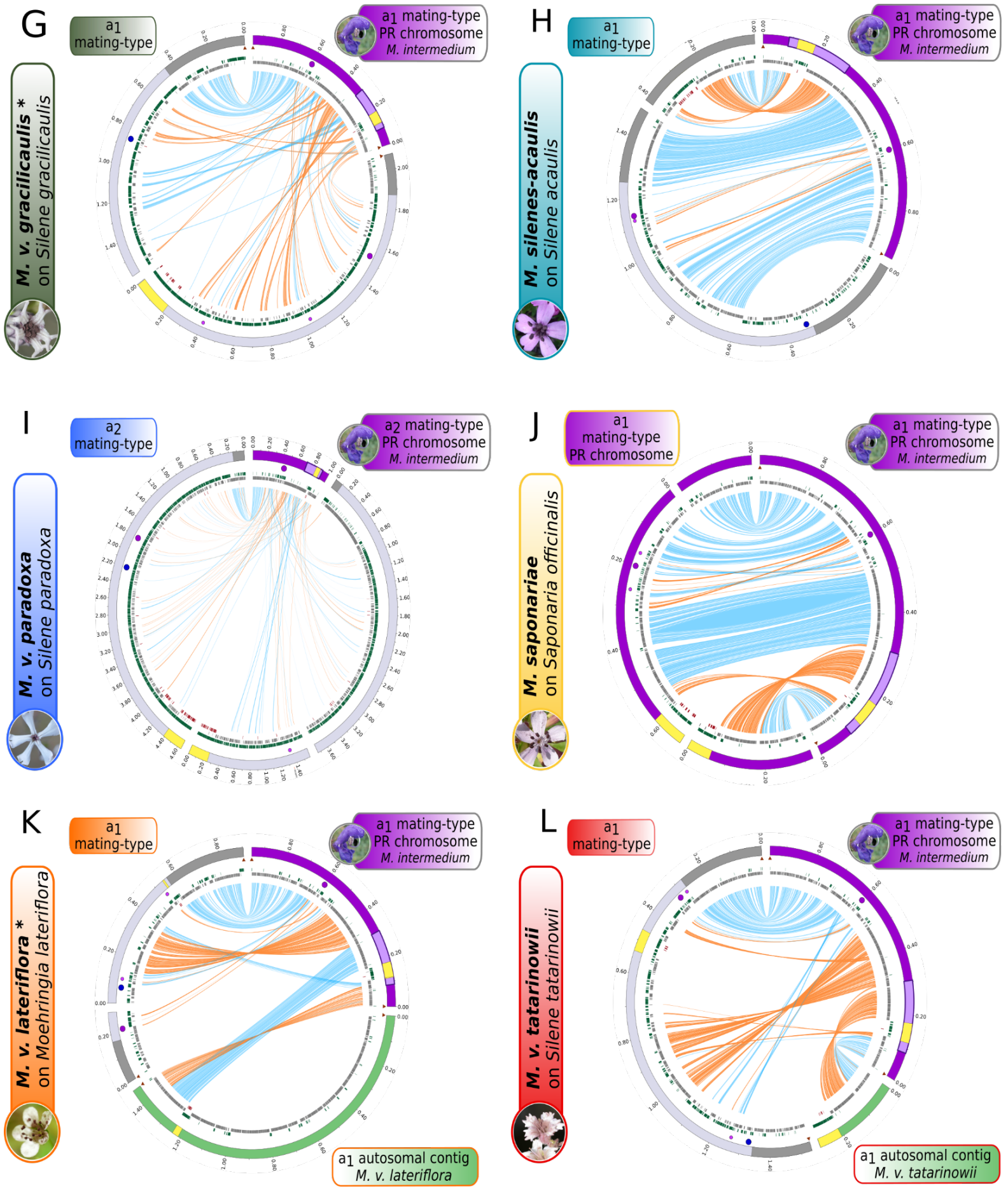

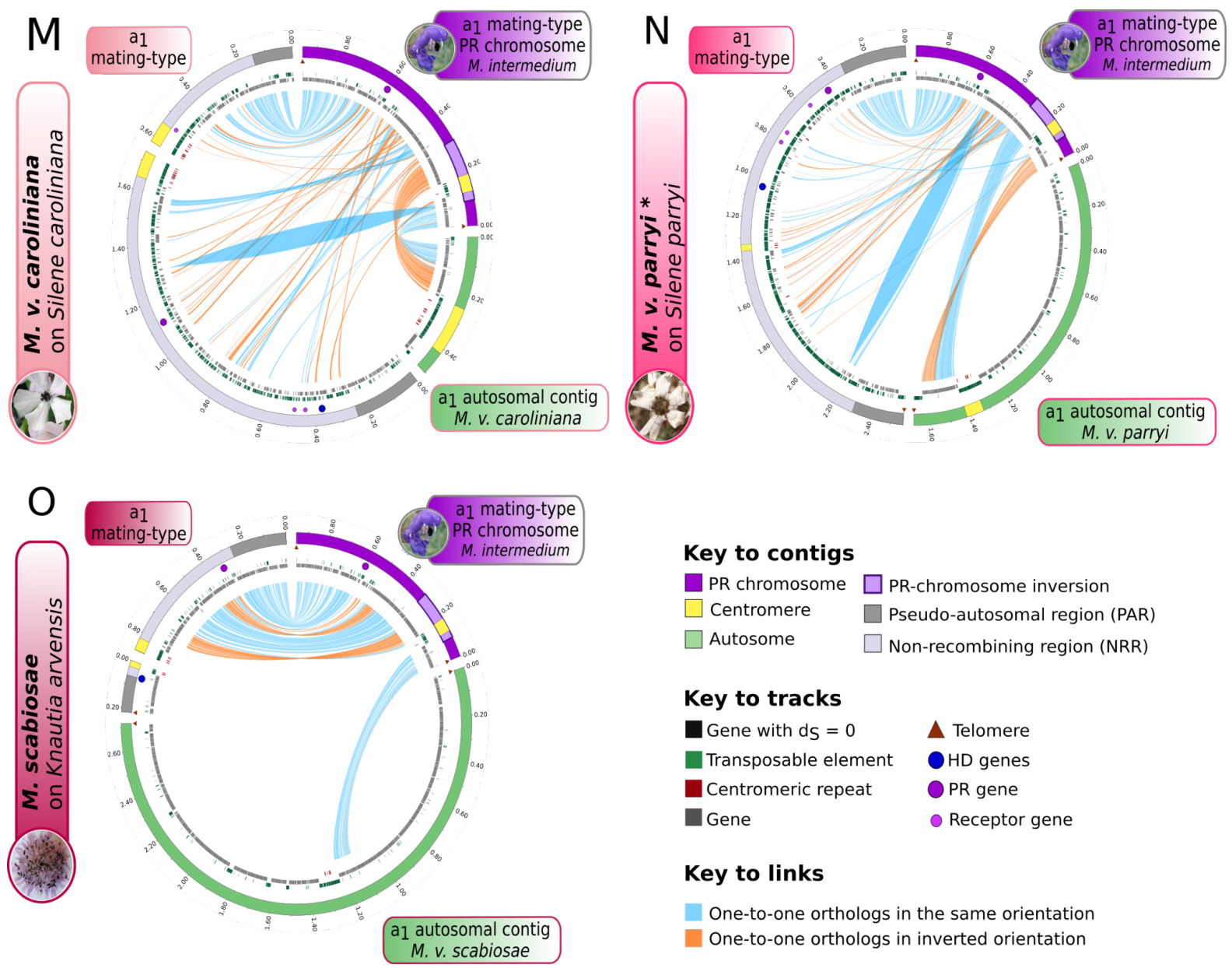

\section{Key to links}

One-to-one orthologs in the same orientation

One-to-one orthologs in inverted orientation

\section{Supplementary figure S3: Inter-specific comparison of gene order between the PR} mating-type chromosome of Microbotryum intermedium and the mating-type chromosomes of all the species with suppressed recombination. Comparison of gene order between $\mathrm{a}_{1}$ mating-type PR chromosome of $M$. intermedium and their homologues on the PRM. coronariae, E) M. v. viscidula, F) M. v. melanantha, G) M. v. gracilicaulis, H) M. silenesacaulis, I) M. v. paradoxa, J) M. saponariae, K) M. v. lateriflora, L) M. v. tatarinowii, M) M. v. caroliniana, N) M. v. parryi and O) M. scabiosae. The PR, HD and pheromone genes are represented by purple, blue and small light purple circles, respectively. The outer track

900 represents contigs, with external ticks every $200 \mathrm{~kb}$. The PR mating-type chromosome of $M$. intermedium is represented in purple. (A to O). The region of the inversion having occurred after divergence with M. scabiosae (Fig. 1), in the region encompassing the centromere of the PR chromosome, is highlighted on the outer track in light purple. The non-recombining region (NRR) and the pseudo-autosomal region (PAR) of the mating-type chromosomes of

905 M. lychnidis-dioicae (A), M. silenes-dioicae (B), M. coronariae (C), M. violaceum s. str. (D), M. v. viscidula (E), M. v. melanantha (F), M. v. gracilicaulis (G), M. silenes-acaulis (H), 
M. v. paradoxa (I), M. saponariae (J), M. v. lateriflora (K), M. v. tatarinowii (L), M. v. caroliniana (M), M. v. parryi (N) and M. scabiosae (O)are represented in light and dark gray, respectively. Green contigs correspond to autosomal contigs, in M. v. lateriflora (K), M. v.

910 tatarinowii (L), M. v. caroliniana (M), M. v. parryi (N) and M. scabiosae (O). The centromeres are represented in yellow. The telomeres are indicated by brown triangles. Black marks on the inner track represent the genes with null synonymous divergence between $a_{1}$ and $\mathrm{a}_{2}$ alleles. Green marks on the inner track represent transposable element (TE) copies. Gray marks on the inner track correspond to genes. Red marks on the inner track correspond 915 to the putative centromeric repeats. Blue and orange lines link alleles, the latter corresponding to inversions. The link width is proportional to the corresponding gene length. 

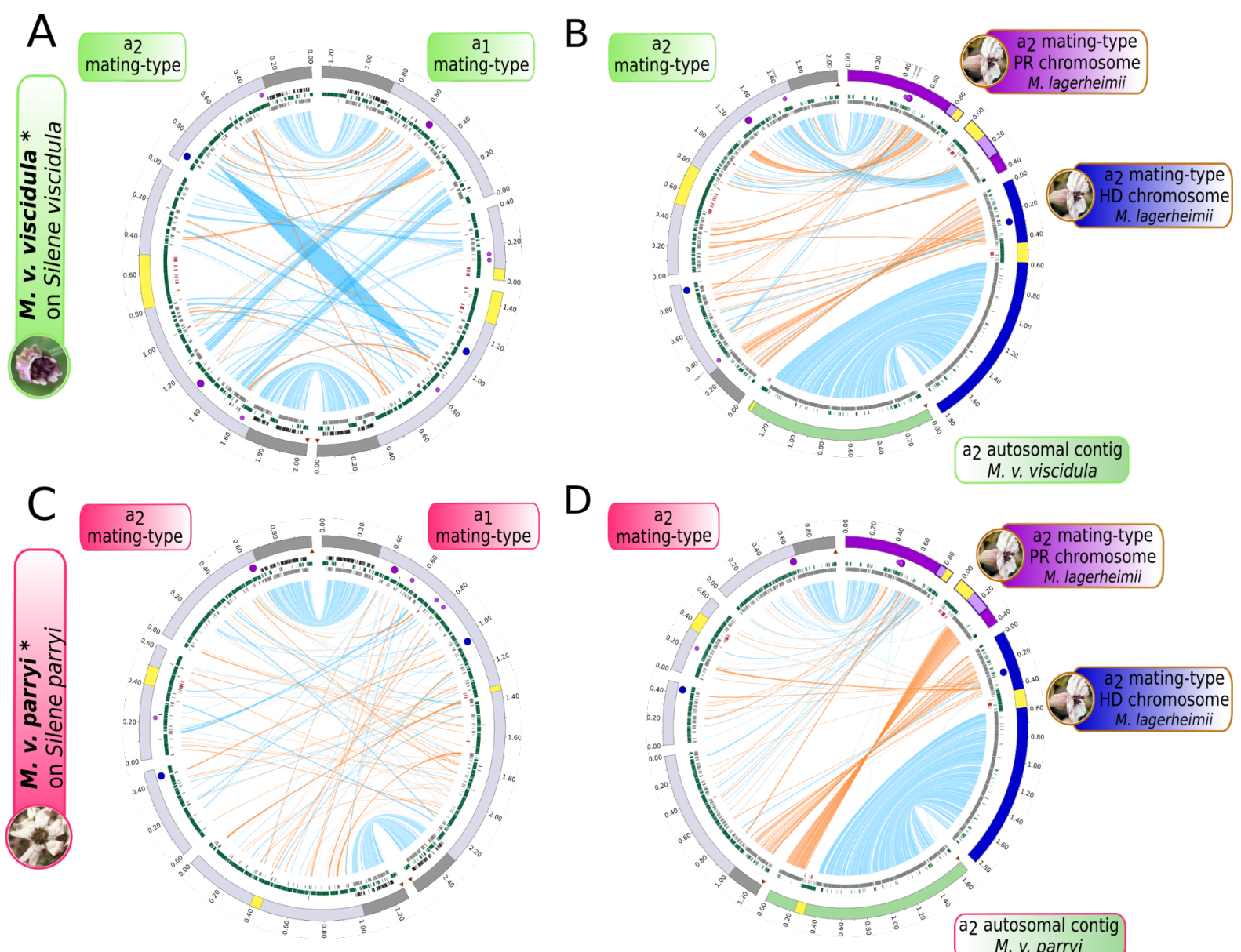

D $\underset{\text { ating-type }}{\text { mats }}$

M. v. viscidula

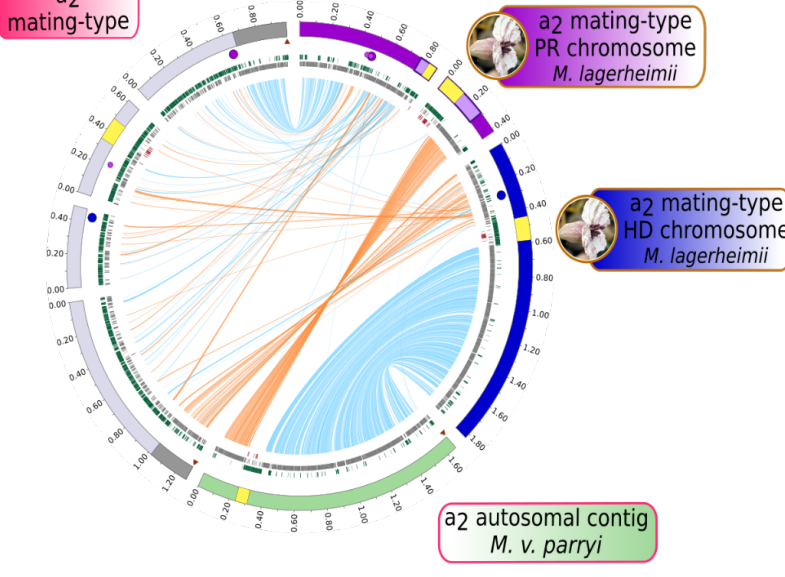

Key to contigs
$\square$ HD chromosome $\square$ PR-chromosome inversion
$\square$ PR chromosome $\square$ Pseudo-autosomal region (PAR)
$\square$ Centromere $\quad \square$ Non-recombining region (NRR)
$\square$ Autosome

Key to tracks

Gene with ds $=0$

Transposable element

Centromeric repeat

Gene
Key to links

One-to-one orthologs in the same orientation

One-to-one orthologs in inverted orientation

\section{Supplementary figure S4: Inter- and intra-specific comparison of gene order between} mating-type chromosomes in Microbotryum violaceum viscidula and M. v. parryi. Synteny plots between A) $\mathrm{a}_{1}$ and $\mathrm{a}_{2}$ mating-type chromosomes of $M$. v. viscidula, B) $\mathrm{a}_{2}$

mating-type chromosome of $M$. lagerheimii and their homologues in $M$. v. viscidula, C) $\mathrm{a}_{1}$ and $\mathrm{a}_{2}$ mating-type chromosomes of $M . v$. parryi, D) $\mathrm{a}_{2}$ mating-type chromosome of $M$. intermedium and their homologues in M. v. parryi. Comparisons of M. lagerheimii $\mathrm{a}_{2}$ HD and PR chromosomes and $M . v$. viscidula (B) and $M$. v. parryi (F) $\mathrm{a}_{2}$ mating-type contigs to infer chromosomal rearrangements having led to HD-PR linkage, considering M. lagerheimii chromosomes as a proxy for the ancestral chromosomal state. The PR, HD and pheromone genes are represented by purple, blue and small light purple circles, respectively. The outer track represents contigs, with length ticks every $200 \mathrm{~kb}$. The HD and PR mating-type chromosomes of $M$. lagerheimii are represented in blue and purple, respectively (B and D). The region of inversion having occurred after divergence with M. scabiosae (Fig. 1), in the 
930 region encompassing the centromere of the PR chromosome, is highlighted in light purple. The non-recombining region (NRR) and the pseudo-autosomal region (PAR) of the matingtype chromosomes of M. v. viscidula (A and B) and M. v. parryi (C and D) are represented in light and dark gray, respectively. Green contigs correspond to autosomal contigs, in $M . v$. viscidula (B) and M. v. parryi (D). The centromeres are represented in yellow. The telomeres 935 are indicated by brown triangles. Black marks on the inner track represent the genes with null synonymous divergence between $\mathrm{a}_{1}$ and $\mathrm{a}_{2}$ alleles. Green marks on the inner track represent transposable element (TE) copies. Gray marks on the inner track correspond to genes. Red marks on the inner track correspond to the putative centromeric repeats. Blue and orange lines link alleles, the latter corresponding to inversions. The link width is proportional to the 940 corresponding gene length. 
A
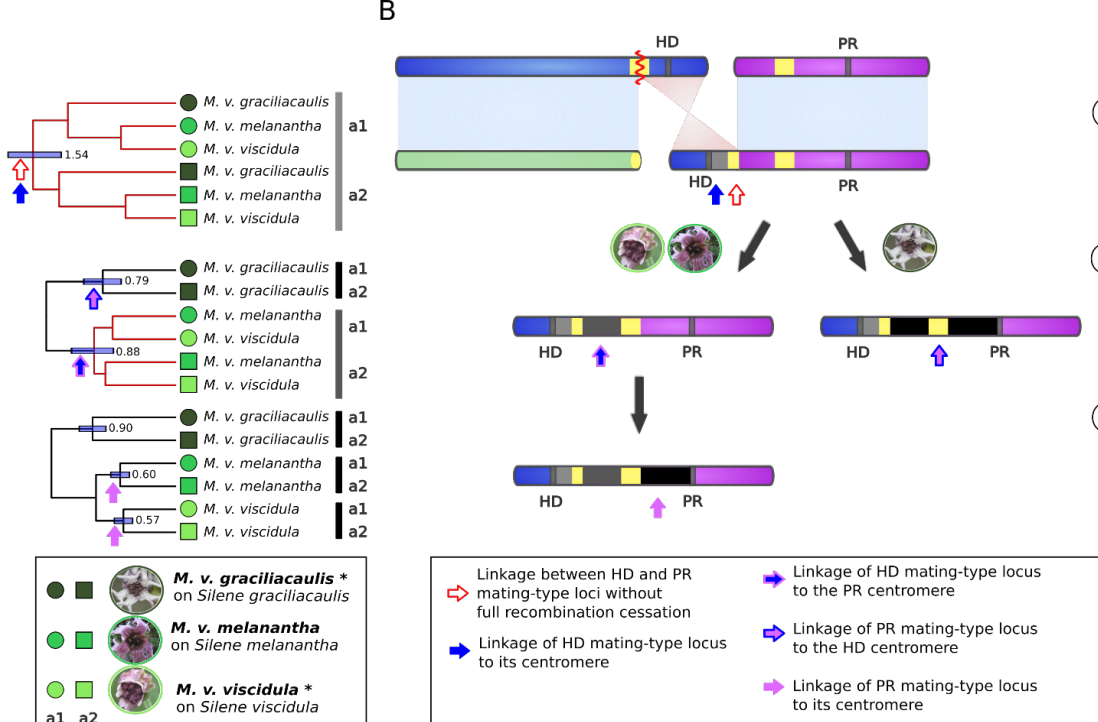

In the ancestor of Microbotryum violaceum viscidula, M. v. melanantha and M. v. graciliacautis:
HD and PR mating-type chromosome fusion an recombination suppression in the light gray stratum ter the first speciation event:

2. Further extension of recombination suppression M. v. melanantha, forming the dark gray stratum ii) to the PR locus in $M$. v. graciliacaulis linking the PR and HD loci and forming the black stratum

Independently in $M$. v. viscidula and $M$. v. melanantha. Recombination suppression between the PR locus and
its centromere linking the PR and HD loci and forming HD the black stratum

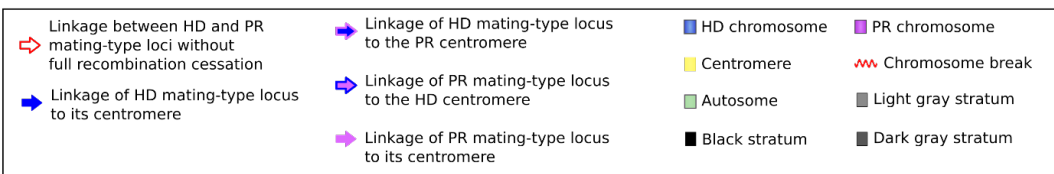

Supplementary figure S5: Reconstructed scenario of stepwise recombination suppression between the HD and PR mating-type loci in the Microbotryum violaceum melanantha clade. A) Timetree of conserved genes in the regions between the HD locus and its ancestral centromere (upper panel), between the short arm telomere and the centromere in 945 the ancestral PR chromosomes (middle panel) or between the PR locus and its ancestral centromere (bottom panel). Topologies are significantly different, showing full trans-specific polymorphism between $\mathrm{a}_{1}$ and $\mathrm{a}_{2}$ alleles (light gray stratum, upper panel), trans-specific polymorphism between $\mathrm{a}_{1}$ and $\mathrm{a}_{2}$ alleles of $M . v$. melanantha and $M . v$. viscidula (dark grey stratum, middle panel) or no trans-specific polymorphism (bottom panel). Trans-specific polymorphisms are indicated by red branches. B) Reconstructed stepwise recombination suppression having generated the light gray, dark grey and black evolutionary strata. Arrows correspond to the suppression of recombination steps 